\title{
Minimization of quotients with variable exponents
}

\author{
C.O. Alves ${ }^{\mathrm{a}}$, M.D.H. Bolaños ${ }^{\mathrm{b}}$, G. Ercole $^{\mathrm{b}}{ }^{*}$ \\ ${ }^{a}$ Universidade Federal de Campina Grande, Campina Grande, PB, 58109-970, Brazil. \\ E-mail: coalves@dme.ufcg.edu.br \\ b Universidade Federal de Minas Gerais, Belo Horizonte, MG, 30.123-970, Brazil. \\ E-mail: emdi_82@hotmail.com \\ E-mail:grey@mat.ufmg.br
}

July 6, 2021

\begin{abstract}
Let $\Omega$ be a bounded domain of $\mathbb{R}^{N}, p \in C^{1}(\bar{\Omega}), q \in C(\bar{\Omega})$ and $l, j \in \mathbb{N}$. We describe the asymptotic behavior of the minimizers of the Rayleigh quotient $\frac{\|\nabla u\|_{l p(x)}}{\|u\|_{j q(x)}}$, first when $j \rightarrow \infty$ and after when $l \rightarrow \infty$.
\end{abstract}

2010 AMS Classification. 35B40; 35J60; 35P30.

Keywords: Asymptotic behavior, infinity Laplacian, variable exponents.

\section{Introduction}

Let $\Omega$ be a bounded domain of $\mathbb{R}^{N}, N \geq 2$, and consider the Rayleigh quotient

$$
\frac{\|\nabla u\|_{p(x)}}{\|u\|_{q(x)}}
$$

associated with the immersion of the Sobolev space $W_{0}^{1, p(x)}(\Omega)$ into the Lebesgue space $L^{q(x)}(\Omega)$, where the variable exponents satisfy

$$
1<\inf _{\Omega} p(x) \leq \sup _{\Omega} p(x)<\infty
$$

and

$$
1<q(x)<p^{*}(x):=\left\{\begin{array}{clll}
\frac{N p(x)}{N-p(x)} & \text { if } & p(x)<N \\
\infty & \text { if } & p(x) \geq N .
\end{array}\right.
$$

${ }^{*}$ Corresponding author 
In this paper we study the behavior of the least Rayleigh quotients when the functions $p(x)$ and $q(x)$ become arbitrarily large. Our script is based on the paper [8], where these functions are constants. Thus, in order to overcome the difficulties imposed by the fact that the exponents depend on $x$, we adapt arguments developed by Franzina and Lindqvist in [18], where $p(x)=q(x)$. Actually, our results in the present paper generalize those of [8] for variable exponents and complement the approach of [18].

In [8], Ercole and Pereira first studied the behavior, when $q \rightarrow \infty$, of the positive minimizers $w_{q}$ corresponding to

$$
\lambda_{q}:=\min \left\{\|\nabla u\|_{L^{p}(\Omega)}: u \in W_{0}^{1, p}(\Omega) \quad \text { in } \quad\|u\|_{L^{q}(\Omega)}=1\right\}
$$

for a fixed $p>N$. An $L^{\infty}$-normalized function $u_{p} \in W_{0}^{1, p}(\Omega)$ is obtained as the uniform limit in $\bar{\Omega}$ of a sequence $w_{q_{n}}$, with $q_{n} \rightarrow \infty$. Such a function is positive in $\Omega$, assumes its maximum only at a point $x_{p}$ and satisfies

$$
\begin{cases}-\Delta_{p} u=\Lambda_{p} \delta_{x_{p}} & \text { in } \Omega \\ u=0 & \text { on } \partial \Omega\end{cases}
$$

where

$$
\Lambda_{p}:=\min \left\{\|\nabla u\|_{L^{p}(\Omega)}: u \in W_{0}^{1, p}(\Omega) \quad \text { in } \quad\|u\|_{L^{\infty}(\Omega)}=1\right\}
$$

and $\delta_{x_{p}}$ denotes the Dirac delta distribution concentrated at $x_{p}$. In the sequence, the behavior of the pair $\left(\Lambda_{p}, u_{p}\right)$, as $p \rightarrow \infty$, is determined. In fact, it is proved that

$$
\lim _{p \rightarrow \infty} \Lambda_{p}=\Lambda_{\infty}:=\inf _{0 \neq v \in W_{0}^{1, \infty}(\Omega)} \frac{\|\nabla v\|_{\infty}}{\|v\|_{\infty}}
$$

and that there exist a sequence $p_{n} \rightarrow \infty$, a point $x_{*} \in \Omega$ and a function $u_{\infty} \in W_{0}^{1, \infty}(\Omega) \cap C(\bar{\Omega})$ such that: $x_{p_{n}} \rightarrow x_{*},\|\rho\|_{\infty}=\rho\left(x_{*}\right), u_{\infty} \leq \frac{\rho}{\|\rho\|_{\infty}}$ and $u_{p_{n}} \rightarrow u_{\infty}$, uniformly in $\bar{\Omega}$. Moreover, it is shown that: $u_{\infty}$ is also a minimizer of $\Lambda_{\infty}$, assumes its maximum value 1 only at $x_{*}$ and satisfies

$$
\begin{cases}\Delta_{\infty} u=0 & \text { in } \Omega \backslash\left\{x_{*}\right\} \\ u=\frac{\rho}{\|\rho\|_{\infty}} & \text { on } \partial\left(\Omega \backslash\left\{x_{*}\right\}\right)=\left\{x_{*}\right\} \cup \partial \Omega\end{cases}
$$

in the viscosity sense.

In [18], Franzina and Lindqvist determined the exact asymptotic behavior, as $j \rightarrow \infty$, of both the minimum $\Lambda_{j p(x)}$ of the quotients $\frac{\|\nabla u\|_{j p(x)}}{\|u\|_{j p(x)}}$ and its respective $j p(x)$-normalized minimizer $u_{j}$. It is proved that

$$
\lim _{j \rightarrow \infty} \Lambda_{j p(x)}=\Lambda_{\infty}
$$

and that a subsequence of $\left(u_{j}\right)_{j \in \mathbb{N}}$ converges uniformly in $\bar{\Omega}$ to a nonnegative function $0 \not \equiv u_{\infty} \in$ $C(\bar{\Omega}) \cap W_{0}^{1, \infty}(\Omega)$ satisfying, in the viscosity sense, the equation

$$
\max \left\{\Lambda_{\infty}-\frac{|\nabla u|}{u} \quad, \quad \Delta_{\infty(x)}\left(\frac{u}{\|\nabla u\|_{\infty}}\right)\right\}=0 \quad \text { in } \Omega
$$


where the operator $\Delta_{\infty(x)}$ is defined by

$$
\Delta_{\infty(x)} u:=\Delta_{\infty} u+|\nabla u|^{2} \ln |\nabla u|\langle\nabla u, \nabla \ln p\rangle .
$$

In the present paper we assume that $p \in C^{1}(\bar{\Omega}), q \in C(\bar{\Omega})$ and $1 \leq q(x)<p^{*}(x)$ in $\bar{\Omega}$. After presenting, in Section 2, a brief review on the theory of Sobolev-Lebesgue spaces with variable exponents, we show in Section 3 that

$$
\Lambda_{1}:=\inf \left\{\frac{\|\nabla v\|_{p(x)}}{\|v\|_{q(x)}}: v \in W_{0}^{1, p(x)}(\Omega) \backslash\{0\}\right\}=\frac{\|\nabla u\|_{p(x)}}{\|u\|_{q(x)}}>0
$$

for some $u \in W_{0}^{1, p(x)}(\Omega) \backslash\{0\}$. Moreover, taking [18] and [25] as reference, we derive the following Euler-Lagrange equation corresponding to this minimization problem

$$
-\operatorname{div}\left(\left|\frac{\nabla u}{K(u)}\right|^{p(x)-2} \frac{\nabla u}{K(u)}\right)=\Lambda S(u)\left|\frac{u}{k(u)}\right|^{q(x)-2} \frac{u}{k(u)},
$$

where $\Lambda=\Lambda_{1}$,

$$
K(u):=\|\nabla u\|_{p(x)}, \quad k(u):=\|u\|_{q(x)}, \quad \text { and } \quad S(u):=\frac{\int_{\Omega}\left|\frac{\nabla u}{K(u)}\right|^{p(x)} \mathrm{d} x}{\int_{\Omega}\left|\frac{u}{k(u)}\right|^{q(x)} \mathrm{d} x} .
$$

We consider (2)-(3) as an eigenvalue problem. Thus, if a pair $(\Lambda, u) \in \mathbb{R} \times W_{0}^{1, p(x)}(\Omega) \backslash\{0\}$ solves (2)-(3) we say that $\Lambda$ is an eigenvalue and $u$ is an eigenfunction corresponding to $\Lambda$. In this setting, $\Lambda_{1}$ is the first eigenvalue and any of its corresponding eigenfunctions is a first eigenfunction. We show that any first eigenfunction do not change sign in $\Omega$ and, for the sake of completeness, we apply a minimax scheme based on Kranoselskii genus to obtain an increasing and unbounded sequence of eigenvalues.

Our main results are established in Sections 4 and 5. First we consider a natural $l>N$ and show, in Section 4, that

$$
\mu_{l}:=\inf \left\{\frac{\|\nabla v\|_{l p(x)}}{\|v\|_{\infty}}: v \in W_{0}^{1, l p(x)}(\Omega) \backslash\{0\}\right\}=\lim _{j \rightarrow \infty} \Lambda_{l, j},
$$

where

$$
\Lambda_{l, j}:=\inf \left\{\frac{\|\nabla v\|_{l p(x)}}{\|v\|_{j q(x)}}: v \in W_{0}^{1, l p(x)}(\Omega) \backslash\{0\}\right\} .
$$

Moreover, by using the results of Section 3, we argue that for each fixed $j>1$ there exists a positive minimizer $u_{l, j} \in W_{0}^{1, l p(x)}(\Omega) \backslash\{0\}$ for $\Lambda_{l, j}$. Hence, the compactness of the embedding $W_{0}^{1, l p(x)}(\Omega) \hookrightarrow C(\bar{\Omega})$ implies that $\mu_{l}$ is achieved at a function $w_{l}$ which is obtained as the uniform limit of $u_{l, j_{m}}$ for a subsequence $j_{m} \rightarrow \infty$. 
We also show in Section 4, by using arguments developed in [19], that $\mu_{l}$ is achieved at $u$ if, and only if,

$$
-\operatorname{div}\left(\left|\frac{\nabla u}{K_{l}(u)}\right|^{l p(x)-2} \frac{\nabla u}{K_{l}(u)}\right)=\mu_{l}\left(\int_{\Omega}\left|\frac{\nabla u}{K_{l}(u)}\right|^{l p(x)} \mathrm{d} x\right) \operatorname{sgn}\left(u\left(x_{0}\right)\right) \delta_{x_{0}},
$$

where $K_{l}(u)=\|\nabla u\|_{l p(x)}$ and $x_{0}$ is the only point where $u$ reaches its uniform norm.

Finally, in Section 5, we study the asymptotic behavior of $\mu_{l}$ and of its normalized extremal function $w_{l}\left(\left\|w_{l}\right\|_{\infty}=1\right.$ and $\left.\mu_{l}=\left\|\nabla w_{l}\right\|_{l p(x)}\right)$, when $l \rightarrow \infty$. We prove that

$$
\lim _{l \rightarrow \infty} \mu_{l}=\Lambda_{\infty}
$$

and that there exist $l_{n} \rightarrow \infty, x_{\star} \in \Omega$ and $w_{\infty} \in W_{0}^{1, \infty}(\Omega) \cap C(\bar{\Omega})$ such that $w_{l_{n}} \rightarrow w_{\infty}$ uniformly in $\bar{\Omega}$ and

$$
0 \leq w_{\infty} \leq \frac{d}{\|d\|_{\infty}} \quad \text { a.e. } \Omega \text { and } \quad w_{\infty}\left(x_{\star}\right)=\frac{d\left(x_{\star}\right)}{\|d\|_{\infty}},
$$

where $d(x)=\operatorname{dist}(x, \partial \Omega)$ is the function distance to the boundary. It is well-known that $d \in$ $W_{0}^{1, \infty}(\Omega)$ and

$$
\Lambda_{\infty}=\frac{1}{\|d\|_{\infty}}
$$

Moreover, we prove that $\Lambda_{\infty}$ is attained at $w_{\infty}$ and that this function satisfies

$$
\begin{cases}\Delta_{\infty(x)}\left(\frac{u}{\|\nabla u\|_{\infty}}\right)=0 & \text { in } \quad D=\Omega \backslash\left\{x_{\star}\right\} \\ \frac{u}{\|\nabla u\|_{\infty}}=d & \text { on } \quad \partial D=\partial \Omega \cup\left\{x_{\star}\right\} .\end{cases}
$$

in the viscosity sense.

Due to the lack of a suitable version of the Harnack's inequality for the "variable infinity operator" $\Delta_{\infty(x)}$, one cannot guarantee that the function $w_{\infty}$ is strictly positive in $\Omega$.

At the end of Section 5, by using a uniqueness result proved in [21] for the equation $\Delta_{\infty(x)} u=$ 0 , we provide a sufficient condition on $\Omega$ for the equality

$$
w_{\infty}=\frac{d}{\|d\|_{\infty}} \text { in } \bar{\Omega}
$$

to hold.

After comparing our results with those of [18], it is interesting to remark that the minimum of the quotients $\frac{\|\nabla u\|_{l p(x)}}{\|u\|_{j q(x)}}$ converges to $\Lambda_{\infty}$ independently of how $l p(x)$ and $j q(x)$ go to $\infty$ : if either $l=j \rightarrow \infty$ in the case $p(x)=q(x)$ or $j \rightarrow \infty$ firstly and then $l \rightarrow \infty$. However, the same do not hold for the corresponding minimizers (or for their respective limit problems). The distinction seems to be due to the Dirac delta that appears in the right-hand term of the Euler-Lagrange equation (2) when $q(x)$ is replaced by $j q(x)$ and $j$ is taken to infinity. The same distinction appears when $p$ and $q$ are constant, as one can check from [8] and [20]. 


\section{Preliminaries}

In this section we recall some definitions and results on the Sobolev-Lebesgue spaces with variable exponents.

Let $\Omega$ be a bounded domain in $\mathbb{R}^{N}$ and $p \in C(\bar{\Omega})$ such that $1<p^{-}:=\inf p(x) \leq p^{+}:=$ $\sup p(x)<\infty$. Let $L^{p(x)}(\Omega)$ denote the space of the Lebesgue measurable functions $u: \Omega \rightarrow \mathbb{R}$ such that

$$
\int_{\Omega}|u|^{p(x)} \mathrm{d} x<\infty
$$

endowed with the Luxemburg norm

$$
\|u\|_{p(x)}=\inf \left\{\gamma>0: \int_{\Omega}\left|\frac{u(x)}{\gamma}\right|^{p(x)} \frac{\mathrm{d} x}{p(x)} \leq 1\right\} .
$$

Note that (4) is equivalent to the norm

$$
|u|_{p(x)}=\inf \left\{\gamma>0: \int_{\Omega}\left|\frac{u(x)}{\gamma}\right|^{p(x)} \mathrm{d} x \leq 1\right\}
$$

introduced by [7] and [16]. In fact, we have

$$
\frac{1}{p^{+}}|u|_{p(x)} \leq\|u\|_{p(x)} \leq|u|_{p(x)}, \quad \forall u \in L^{p(x)}(\Omega) .
$$

An important concept in the theory of spaces $L^{p(x)}(\Omega)$ is the modular function.

Definition 2.1 The function $\rho: L^{p(x)}(\Omega) \rightarrow \mathbb{R}$ defined by

$$
\rho(u)=\int_{\Omega}|u|^{p(x)} \frac{\mathrm{d} x}{p(x)},
$$

is called the modular function associated to the space $L^{p(x)}(\Omega)$.

The following proposition lists some properties of the modular function .

Proposition 2.2 Let $u \in L^{p(x)}(\Omega) \backslash\{0\}$, then

a) $\|u\|_{p(x)}=a$ if, and only if, $\rho\left(\frac{u}{a}\right)=1$;

b) $\|u\|_{p(x)}<1(=1 ;>1)$ if, and only if, $\rho(u)<1(=1 ;>1)$;

c) If $\|u\|_{p(x)}>1$ then $\|u\|_{p(x)}<\|u\|_{p(x)}^{p^{-}} \leq \rho(u) \leq\|u\|_{p(x)}^{p^{+}}$;

d) If $\|u\|_{p(x)}<1$ then $\|u\|_{p(x)}^{p^{+}} \leq \rho(u) \leq\|u\|_{p(x)}^{p^{-}}<\|u\|_{p(x)}$. 
For a posterior use, we recall the following estimate valid for an arbitrary $u \in L^{\infty}(\Omega)$ :

$$
\|u\|_{p(x)} \leq \alpha\|u\|_{\infty}, \quad \text { where } \quad \alpha:=\left\{\begin{array}{lll}
|\Omega|^{1 / p^{+}} & \text {if } & |\Omega| \leq 1 \\
|\Omega|^{1 / p^{-}} & \text {if } & |\Omega|>1 .
\end{array}\right.
$$

This estimate is easily verified by applying item b) of Proposition 2.2 to the function $\frac{u}{\alpha\|u\|_{\infty}}$.

We define the Sobolev space

$$
W^{1, p(x)}(\Omega):=\left\{u \in L^{p(x)}(\Omega):|\nabla u| \in L^{p(x)}(\Omega)\right\},
$$

endowed with the norm

$$
\|u\|_{1, p(x)}:=\|u\|_{p(x)}+\|\nabla u\|_{p(x)} .
$$

Both $\left(L^{p(x)}(\Omega),\|\cdot\|_{p(x)}\right)$ and $\left(W^{1, p(x)}(\Omega),\|\cdot\|_{1, p(x)}\right)$ are separable and uniformly convex (therefore, reflexive) Banach spaces.

The Sobolev space $W_{0}^{1, p(x)}(\Omega)$ is defined as the closure of $C_{0}^{\infty}(\Omega)$ in $W^{1, p(x)}(\Omega)$. In this space, $\|\nabla \cdot\|_{p(x)}$ is a norm equivalent to norm $\|\cdot\|_{1, p(x)}$ and this is a consequence of the following proposition.

Proposition 2.3 (see [16]) Let $p \in C(\bar{\Omega})$ with $p^{-}>1$. There exists a positive constant $C$ such that

$$
\|u\|_{p(x)} \leq C\|\nabla u\|_{p(x)}, \quad \forall u \in W_{0}^{1, p(x)}(\Omega) .
$$

Now, we recall some facts involving exponents $q(x) \leq p(x)$.

Proposition 2.4 (see [16]) Let $p, q \in C(\bar{\Omega})$. Then

$$
L^{p(x)}(\Omega) \subset L^{q(x)}(\Omega)
$$

if, and only if, $q(x) \leq p(x)$ in $\Omega$. Additionally, the embedding is continuous.

From now on, the notation $f \ll g$ will mean that $f(x) \leq g(x)$ for all $x \in \bar{\Omega}$ and

$$
\inf _{\Omega}(g(x)-f(x))>0 .
$$

Proposition 2.5 ( [13], [16]) Let $p, q \in C(\bar{\Omega})$ and $1 \leq q(x) \leq p^{*}(x)$ in $\bar{\Omega}$. The embedding

$$
W_{0}^{1, p(x)}(\Omega) \hookrightarrow L^{q(x)}(\Omega)
$$

is continuous. Moreover, it is compact whenever $q \ll p^{\star}$.

We define the operator $p(x)$-Laplacian by $\Delta_{p(x)} u:=\operatorname{div}\left(|\nabla u|^{p(x)-2} \nabla u\right)$ and consider the Dirichlet problem

$$
\begin{cases}-\Delta_{p(x)} u=f(x, u), & x \in \Omega \\ u=0 & x \in \partial \Omega\end{cases}
$$

where $f \in C(\bar{\Omega} \times \mathbb{R}, \mathbb{R})$.

We say that a function $u \in W_{0}^{1, p(x)}(\Omega)$ is a weak solution of (7) if, and only if,

$$
\int_{\Omega}|\nabla u|^{p(x)-2} \nabla u \cdot \nabla \eta \mathrm{d} x=\int_{\Omega} f(x, u) \eta \mathrm{d} x, \quad \forall \eta \in W_{0}^{1, p(x)}(\Omega) .
$$


Proposition 2.6 Weak solutions of (1) belong to $L^{\infty}(\Omega)$ provided that $f$ satisfies the sub-critical growth condition

$$
|f(x, t)| \leq c_{1}+c_{2}|t|^{\alpha(x)-1}, \quad \forall(x, t) \in \Omega \times \mathbb{R},
$$

where $\alpha \in C(\bar{\Omega})$ and $1<\alpha \ll p^{*}$.

Proposition 2.7 Suppose that $p(x)$ is Hölder continuous on $\bar{\Omega}$. If $u \in W_{0}^{1, p(x)}(\Omega) \cap L^{\infty}(\Omega)$ is a weak solution of (17), then $u \in C^{1, \tau}(\bar{\Omega})$ for some $\tau \in(0,1)$.

The following strong maximum principle for $p(x)$-Laplacian is taken from [11].

Proposition 2.8 Suppose that $p \in C^{1}(\bar{\Omega}), u \in W_{0}^{1, p(x)}(\Omega) \backslash\{0\}$ and $u \geq 0$ in $\Omega$. If $-\Delta_{p(x)} u \geq 0$ in $\Omega$ then $u>0$ in $\Omega$.

We recall that the inequality $-\Delta_{p(x)} u \geq 0$, for a function $u \in W_{0}^{1, p(x)}(\Omega)$, means

$$
\int_{\Omega}|\nabla u|^{p(x)-2} \nabla u \cdot \nabla \eta \mathrm{d} x \geq 0, \quad \forall \eta \in W_{0}^{1, p(x)}(\Omega), \text { with } \eta \geq 0 .
$$

Theoretical results involving operators with variable exponent can be found among the papers 2, 7, 10 18, 23 27, 29] and in the references therein. For applications in rheology and image restoration we refer the reader to [1, 3, 28] and [5, 6], respectively.

\section{The minimization problem}

In this section we will consider $p \in C^{1}(\bar{\Omega})$ and $q \in C(\bar{\Omega})$, with $1 \leq q \ll p^{*}$. For practical purposes, $X$ will denote the Sobolev space $W_{0}^{1, p(x)}(\Omega)$ and $k, K: X \rightarrow \mathbb{R}$ will denote, respectively, the functionals

$$
k(u):=\|u\|_{q(x)} \quad \text { and } \quad K(u):=\|\nabla u\|_{p(x)}, \quad u \in X .
$$

Since $K(u)=\|u\|_{X}$, the functional $K$ is sequentially weakly lower semicontinuous in $X$.

We will also consider

$$
\Lambda_{1}:=\inf _{v \in X \backslash\{0\}} \frac{K(v)}{k(v)}
$$

which is positive number, according to Proposition 2.5.

We say that a function $u \in X \backslash\{0\}$ is an extremal function (or minimizer) of $\Lambda_{1}$ if

$$
\frac{K(u)}{k(u)}=\Lambda_{1}
$$

The next proposition shows that such a function always exists.

Proposition 3.1 There exists a nonnegative extremal function of $\Lambda_{1}$. 
Proof. Let $\left(v_{n}\right) \subset X \backslash\{0\}$ be a minimizing sequence of admissible functions such that $k\left(v_{n}\right)=1$. Thus,

$$
\Lambda_{1}=\lim _{n \rightarrow \infty} K\left(v_{n}\right) .
$$

Since the sequence $\left(v_{n}\right)$ is bounded in the reflexive space $X$, there exist a subsequence $\left(v_{n_{j}}\right)$ and $u \in X$ such that $v_{n_{j}} \rightarrow u$ in $X$. We can assume, from Proposition 2.5, that $v_{n_{j}} \rightarrow u$ in $L^{q(x)}(\Omega)$, so that $k\left(v_{n_{j}}\right) \rightarrow k(u)=1$. Since $\|u\|_{X} \leq \liminf _{j}\left\|v_{n_{j}}\right\|_{X}$ we have

$$
K(u) \leq \lim _{j \rightarrow \infty} K\left(v_{n_{j}}\right)=\Lambda_{1}
$$

showing thus that $u$ is an extremal function of $\Lambda_{1}$. It is simple to see that (the nonnegative) function $|u|$ is also an extremal function of $\Lambda_{1}$.

Our next goal is to derive the Euler-Lagrange equation associated with the minimizing problem (9), which must be satisfied for the extremal functions of $\Lambda_{1}$. For this we need the following lemma.

Lemma 3.2 (Lemma A.1, [18]) Let $u \in X$ and $\eta \in C_{0}^{\infty}(\Omega)$. Then,

$$
\left.\frac{d}{d \varepsilon} K(u+\varepsilon \eta)\right|_{\varepsilon=0}=\frac{\int_{\Omega}\left|\frac{\nabla u}{K(u)}\right|^{p(x)-2} \frac{\nabla u}{K(u)} \cdot \nabla \eta \mathrm{d} x}{\int_{\Omega}\left|\frac{\nabla u}{K(u)}\right|^{p(x)} \mathrm{d} x}
$$

and

$$
\left.\frac{d}{d \varepsilon} k(u+\varepsilon \eta)\right|_{\varepsilon=0}=\frac{\int_{\Omega}\left|\frac{u}{k(u)}\right|^{q(x)-2} \frac{u}{k(u)} \eta \mathrm{d} x}{\int_{\Omega}\left|\frac{u}{k(u)}\right|^{q(x)} \mathrm{d} x}
$$

We observe that a necessary condition for the inequality

$$
\frac{K(u)}{k(u)} \leq \frac{K(u+\varepsilon \eta)}{k(u+\varepsilon \eta)}
$$

to hold is that

$$
\left.\frac{d}{d \varepsilon} \frac{K(u+\varepsilon \eta)}{k(u+\varepsilon \eta)}\right|_{\varepsilon=0}=0
$$

which can be written as

$$
\left.\frac{1}{K(u)} \frac{d}{d \varepsilon} K(u+\varepsilon \eta)\right|_{\varepsilon=0}=\left.\frac{1}{k(u)} \frac{d}{d \varepsilon} k(u+\varepsilon \eta)\right|_{\varepsilon=0} .
$$

Therefore, according to Lemma 3.2, if $u$ is an extremal function, then one must have

$$
\int_{\Omega}\left|\frac{\nabla u}{K(u)}\right|^{p(x)-2} \frac{\nabla u}{K(u)} \cdot \nabla \eta \mathrm{d} x=\Lambda_{1} S(u) \int_{\Omega}\left|\frac{u}{k(u)}\right|^{q(x)-2} \frac{u}{k(u)} \eta \mathrm{d} x, \quad \forall \eta \in C_{0}^{\infty}(\Omega)
$$


where

$$
S(u):=\frac{\int_{\Omega}\left|\frac{\nabla u}{K(u)}\right|^{p(x)} \mathrm{d} x}{\int_{\Omega}\left|\frac{u}{k(u)}\right|^{q(x)} \mathrm{d} x} .
$$

Hence, since $X$ is the closure of $C_{0}^{\infty}(\Omega)$ in the norm $\|\cdot\|_{X}$, the Euler-Lagrange equation for the extremal functions is

$$
-\operatorname{div}\left(\left|\frac{\nabla u}{K(u)}\right|^{p(x)-2} \frac{\nabla u}{K(u)}\right)=\Lambda_{1} S(u)\left|\frac{u}{k(u)}\right|^{q(x)-2} \frac{u}{k(u)} .
$$

Definition 3.3 We say that a real number $\Lambda$ is an eigenvalue if there exists $u \in X \backslash\{0\}$ such that

$$
\int_{\Omega}\left|\frac{\nabla u}{K(u)}\right|^{p(x)-2} \frac{\nabla u}{K(u)} \cdot \nabla \eta \mathrm{d} x=\Lambda S(u) \int_{\Omega}\left|\frac{u}{k(u)}\right|^{q(x)-2} \frac{u}{k(u)} \eta \mathrm{d} x, \quad \forall \eta \in X .
$$

In this case, we say that $u$ is an eigenfunction corresponding to $\Lambda$.

Remark 3.4 One can easily verify the following homogeneity property: if $u$ is an eigenfunction corresponding to $\Lambda$ the same holds for tu, for any $t \in \mathbb{R} \backslash\{0\}$.

Taking $\eta=u$ in (12) and recalling the definition of $S(u)$ in (10) we obtain

$$
K(u) \int_{\Omega}\left|\frac{\nabla u}{K(u)}\right|^{p(x)} \mathrm{d} x=\Lambda S(u) k(u) \int_{\Omega}\left|\frac{u}{k(u)}\right|^{q(x)} \mathrm{d} x=\Lambda k(u) \int_{\Omega}\left|\frac{\nabla u}{K(u)}\right|^{p(x)} \mathrm{d} x,
$$

so that

$$
\Lambda=\frac{K(u)}{k(u)} \geq \Lambda_{1}
$$

Hence, $\Lambda_{1}$ is called the first eigenvalue and the corresponding eigenfunctions are called first eigenfunctions. Clearly, the extremal functions are precisely the first eigenfunctions.

Proposition 3.5 There exists a continuous, strictly positive first eigenfunction.

Proof. Proposition 3.1 shows that a nonnegative first eigenfunction $u \in X$ exists, Propositions 2.6 and 2.7 guarantee that $u \in C(\bar{\Omega})$ and the strong maximum principle (Proposition 2.8) yields that $u>0$ in $\Omega$.

Remark 3.6 It can be verified that if the norm (5) is taken to define

$$
\widetilde{\Lambda}_{1}:=\inf _{v \in X \backslash\{0\}} \frac{|\nabla v|_{p(x)}}{|v|_{q(x)}}
$$

then

$$
\frac{1}{p^{+}} \widetilde{\Lambda}_{1} \leq \Lambda_{1} \leq q^{+} \widetilde{\Lambda}_{1}
$$


Moreover, the same results of Propositions 3.1 and 3.5 can be obtained, but associated with an Euler-Lagrange equation a bit more complicated:

$$
\int_{\Omega} p(x)\left|\frac{\nabla u}{K_{\star}}\right|^{p(x)-2} \frac{\nabla u}{K_{\star}} \cdot \nabla \eta \mathrm{d} x=\frac{K_{\star}}{k_{\star}} S_{u} \int_{\Omega} q(x)\left|\frac{u}{k_{\star}}\right|^{q(x)-2} \frac{u}{k_{\star}} \eta \mathrm{d} x
$$

where,

$$
K_{\star}=|\nabla u|_{p(x)}, \quad k_{\star}=|u|_{q(x)}, \quad S_{u}=\frac{\int_{\Omega} p(x)\left|\frac{\nabla u}{K_{\star}}\right|^{p(x)} \mathrm{d} x}{\int_{\Omega} q(x)\left|\frac{u}{k_{\star}}\right|^{q(x)} \mathrm{d} x} .
$$

Noting that $\frac{p^{-}}{q^{+}} \leq S_{u} \leq \frac{p^{+}}{q^{-}}$we can show the existence of a strictly positive eigenfunction. In fact, if $u$ is a nonnegative function of (13) then, for any $\eta \in W_{0}^{1, p(x)}(\Omega)$, with $\eta \geq 0$, we have

$$
\begin{aligned}
p^{+} \int_{\Omega}\left|\frac{\nabla u}{K_{\star}}\right|^{p(x)-2} \frac{\nabla u}{K_{\star}} \cdot \nabla \eta \mathrm{d} x & \geq q^{-} \frac{K_{\star}}{k_{\star}} S_{u} \int_{\Omega}\left|\frac{u}{k_{\star}}\right|^{q(x)-2} \frac{u}{k_{\star}} \eta \mathrm{d} x \\
& \geq q^{-}\left(\frac{p^{-}}{q^{+}}\right) \frac{K_{\star}}{k_{\star}} \int_{\Omega}\left|\frac{u}{k_{\star}}\right|^{q(x)-2} \frac{u}{k_{\star}} \eta \mathrm{d} x \\
& \geq\left(\frac{p^{-}}{q^{+}}\right) \frac{q^{-}}{q^{+}} \frac{K(u)}{k(u)} \int_{\Omega}\left|\frac{u}{k_{\star}}\right|^{q(x)-2} \frac{u}{k_{\star}} \eta \mathrm{d} x .
\end{aligned}
$$

It follows that $-\Delta_{p(x)}\left(\frac{u}{K_{\star}}\right) \geq 0$ what implies, by Proposition 2.8, that $u>0$ in $\Omega$. Thus, we can see that the use of $p(x)^{-1} \mathrm{~d} x$ simplifies the equations a little.

According to Lemma 3.2, the Gateaux derivatives $K^{\prime}, k^{\prime}$ are given, respectively, by

$$
\left\langle K^{\prime}(u), v\right\rangle=\frac{\int_{\Omega}\left|\frac{\nabla u}{K(u)}\right|^{p(x)-2} \frac{\nabla u}{K(u)} \cdot \nabla v \mathrm{~d} x}{\int_{\Omega}\left|\frac{\nabla u}{K(u)}\right|^{p(x)} \mathrm{d} x}, \quad u, v \in X
$$

and

$$
\left\langle k^{\prime}(u), v\right\rangle=\frac{\int_{\Omega}\left|\frac{u}{k(u)}\right|^{q(x)-2} \frac{u}{k(u)} v \mathrm{~d} x}{\int_{\Omega}\left|\frac{u}{k(u)}\right|^{q(x)} \mathrm{d} x}, \quad u, v \in X .
$$

It is simple to check that $K, k \in C^{1}(X, \mathbb{R})$ (see [12,14]). Thus, we define

$$
\mathcal{M}:=\{u \in X: k(u)=1\}=k^{-1}(1)
$$

Since 1 is a regular value of $k$, the set $\mathcal{M}$ is a submanifold of class $C^{1}$ in $X$. The functional

$$
\widetilde{K}:=\left.K\right|_{\mathcal{M}}: \mathcal{M} \rightarrow \mathbb{R}
$$


is of class $C^{1}$ and bounded from below in $\mathcal{M}$.

We know that $u$ is a critical point of $\widetilde{K}$ in $\mathcal{M}$ if there exists $\Lambda \in \mathbb{R}$ such that

$$
K^{\prime}(u)=\Lambda k^{\prime}(u) \text { in } X^{*},
$$

meaning that

$$
\left\langle K^{\prime}(u), v\right\rangle=\Lambda\left\langle k^{\prime}(u), v\right\rangle, \quad \forall v \in X .
$$

Therefore, if $u$ is critical point of $\widetilde{K}$ then $u$ is solution of (12) with $\Lambda=K(u) / k(u)$.

Now, by adapting arguments of [25, Lemma 2.3], we show that $\widetilde{K}$ satisfies the Palais-Smale condition.

Proposition 3.7 $\widetilde{K}$ satisfies the $(P S)_{c}$ condition for all $c \in \mathbb{R}$, namely, every sequence $\left(u_{n}\right) \subset$ $\mathcal{M}$ such that $\widetilde{K}\left(u_{n}\right) \rightarrow c$ and $\widetilde{K}^{\prime}\left(u_{n}\right) \rightarrow 0$, has a convergent subsequence.

Proof. First, we show that if $u \in X \backslash\{0\}$, then

$$
\left|\left\langle K^{\prime}(u), v\right\rangle\right| \leq K(v) \quad \text { and } \quad\left|\left\langle k^{\prime}(u), v\right\rangle\right| \leq k(v), \quad \forall v \in X .
$$

We assume that $v \not \equiv 0$ (otherwise the equality in (14) holds trivially). Then

$$
\left|\left\langle k^{\prime}(u), v\right\rangle\right| \leq \frac{\int_{\Omega}\left|\frac{u}{k(u)}\right|^{q(x)-1}|v| \mathrm{d} x}{\int_{\Omega}\left|\frac{u}{k(u)}\right|^{q(x)} \mathrm{d} x}
$$

and, by using the Young inequality

$$
a b \leq\left(1-\frac{1}{r}\right) a^{r / r-1}+\frac{1}{r} b^{r}, \quad \forall a, b \geq 0, r>1,
$$

with $a=|u / k(u)|^{q(x)-1}, b=|v / k(v)|, r=q(x)$, and integrating over $\Omega$, we have

$$
\int_{\Omega}\left|\frac{u}{k(u)}\right|^{q(x)-1}\left|\frac{v}{k(v)}\right| \mathrm{d} x \leq \int_{\Omega}\left|\frac{u}{k(u)}\right|^{q(x)} \mathrm{d} x-\int_{\Omega}\left|\frac{u}{k(u)}\right|^{q(x)} \frac{\mathrm{d} x}{q(x)}+\int_{\Omega}\left|\frac{v}{k(v)}\right|^{q(x)} \frac{\mathrm{d} x}{q(x)} .
$$

Since

$$
\|u\|_{q(x)}=k(u) \quad \text { and } \quad\|v\|_{q(x)}=k(v)
$$

it follows from Proposition $2.2($ item $a)$ ) that

$$
\int_{\Omega}\left|\frac{u}{k(u)}\right|^{q(x)} \frac{\mathrm{d} x}{q(x)}=1=\int_{\Omega}\left|\frac{v}{k(v)}\right|^{q(x)} \frac{\mathrm{d} x}{q(x)} .
$$

This implies that (16) can be rewritten as

$$
\int_{\Omega}\left|\frac{u}{k(u)}\right|^{q(x)-1}|v| \mathrm{d} x \leq k(v) \int_{\Omega}\left|\frac{u}{k(u)}\right|^{q(x)} \mathrm{d} x
$$


which, in view of (15), leads to the second inequality in (14).

The first inequality in (14) is obtained by using the same arguments.

Now, let $c \in \mathbb{R}$ and take a sequence $\left(u_{n}\right) \subset \mathcal{M}$ such that $\widetilde{K}\left(u_{n}\right) \rightarrow c$ and $\widetilde{K}^{\prime}\left(u_{n}\right) \rightarrow 0$. It follows that $K\left(u_{n}\right) \rightarrow c$ in $X$ and

$$
K^{\prime}\left(u_{n}\right)-c_{n} k^{\prime}\left(u_{n}\right) \rightarrow 0
$$

in $X^{*}$, for some sequence $\left(c_{n}\right) \subset \mathbb{R}$. Since

$$
\left\langle K^{\prime}\left(u_{n}\right)-c_{n} k^{\prime}\left(u_{n}\right), u_{n}\right\rangle=\left\langle K^{\prime}\left(u_{n}\right), u_{n}\right\rangle-c_{n}\left\langle k^{\prime}\left(u_{n}\right), u_{n}\right\rangle=K\left(u_{n}\right)-c_{n}
$$

and

$$
\begin{aligned}
\left\|\left\langle K^{\prime}\left(u_{n}\right)-c_{n} k^{\prime}\left(u_{n}\right), u_{n}\right\rangle\right\|_{X} & \leq\left\|K^{\prime}\left(u_{n}\right)-c_{n} k^{\prime}\left(u_{n}\right)\right\|_{X^{*}}\left\|u_{n}\right\|_{X} \\
& =\left\|K^{\prime}\left(u_{n}\right)-c_{n} k^{\prime}\left(u_{n}\right)\right\|_{X^{*}} K\left(u_{n}\right) \rightarrow 0,
\end{aligned}
$$

one has $c_{n} \rightarrow c$.

Taking into account that $K\left(u_{n}\right)=\left\|u_{n}\right\|_{X}$ is bounded and that $X$ is reflexive and compactly embedded into $L^{q(x)}(\Omega)$, we can select a subsequence $\left(u_{n_{j}}\right)$ converging weakly in $X$ and strongly in $L^{q(x)}(\Omega)$ to a function $u \in X$. The weak convergence guarantees that $K(u) \leq \liminf K\left(u_{n_{j}}\right)$. Thus, since $X$ is uniformly convex, in order to conclude that $u_{n_{j}}$ converges to $u$ strongly, it is enough to verify that

$$
\limsup _{j \rightarrow \infty} K\left(u_{n_{j}}\right) \leq K(u) .
$$

It follows from (14) that

$$
\left|\left\langle k^{\prime}\left(u_{n_{j}}\right), u_{n_{j}}-u\right\rangle\right| \leq k\left(u_{n_{j}}-u\right)=\left\|u_{n_{j}}-u\right\|_{q(x)} \rightarrow 0 .
$$

Combining this fact, (17) and the boundedness of both sequences $\left(c_{n_{j}}\right)$ and $\left(\left\|u_{n_{j}}-u\right\|_{X}\right)$ we conclude that $\left\langle K^{\prime}\left(u_{n_{j}}\right), u_{n_{j}}-u\right\rangle \rightarrow 0$. Since

$$
\left\langle K^{\prime}\left(u_{n_{j}}\right), u_{n_{j}}-u\right\rangle=K\left(u_{n_{j}}\right)-\left\langle K^{\prime}\left(u_{n_{j}}\right), u\right\rangle \geq K\left(u_{n_{j}}\right)-K(u)
$$

we have

$$
\limsup _{j \rightarrow \infty} K\left(u_{n_{j}}\right) \leq \limsup _{j \rightarrow \infty}\left\langle K^{\prime}\left(u_{n_{j}}\right), u_{n_{j}}-u\right\rangle+K(u)=K(u),
$$

what finishes the proof.

Since $\mathcal{M}$ is a closed symmetric submanifold of class $C^{1}$ in $X$ and $\widetilde{K} \in C^{1}(\mathcal{M}, \mathbb{R})$ is even, bounded from below and satisfies the $(P S)_{c}$ condition, we can define an increasing and unbounded sequence of eigenvalues, by a minimax scheme. For this, we set

$$
\Sigma:=\{A \subset X \backslash\{0\}: A \text { is compact and } A=-A\}
$$

and

$$
\Sigma_{n}:=\{A \in \Sigma: A \subset \mathcal{M} \text { and } \gamma(A) \geq n\}, \quad n=1,2, \ldots,
$$


where $\gamma$ is the Krasnoselskii genus.

Let us define

$$
\lambda_{n}:=\inf _{A \in \Sigma_{n}} \sup _{u \in A} \widetilde{K}(u), \quad n \geq 1 .
$$

It is known that under the above conditions for $\mathcal{M}$ and $\widetilde{K}$, we have that $\lambda_{n}$ is a critical value of $\widetilde{K}$ in $\mathcal{M}$ (see [30], Corollary 4.1). Moreover, since $\Sigma_{k+1} \subset \Sigma_{k}$, we have $\lambda_{k+1} \geq \lambda_{k}$, and so

$$
0<\lambda_{1} \leq \lambda_{2} \leq \ldots \leq \lambda_{n} \leq \lambda_{n+1} \ldots \rightarrow \infty .
$$

In particular $\lambda_{1}=\inf _{v \in \mathcal{M}} K(v)=\Lambda_{1}$ (this latter equality is consequence of Remark 3.4).

Let us consider the sets

$$
Q_{n}=\left\{u \in \mathcal{M}: \widetilde{K}^{\prime}(u)=0 \text { and } \quad \widetilde{K}(u)=\lambda_{n}\right\}
$$

and

$$
A_{p(x), q(x)}=\{\lambda \in \mathbb{R}: \lambda \text { is an eigenvalue }\} .
$$

If $u \in Q_{n}$, for a given $n \geq 1$, there exists $\Lambda \in \mathbb{R}$ such that $K^{\prime}(u)=\Lambda k^{\prime}(u)$ in $X^{*}$, i.e., $\left\langle K^{\prime}(u), v\right\rangle=$ $\Lambda\left\langle k^{\prime}(u), v\right\rangle$ for any $v \in X$. Thus, $\Lambda=\frac{K(u)}{k(u)}=\lambda_{n}$, so that $\left(\lambda_{n}\right)_{n \geq 1} \subset A_{p(x), q(x)}$. Since $\lambda_{n} \rightarrow \infty$, we conclude the following.

Proposition 3.8 The set of eigenvalues $A_{p(x), q(x)}$ is non-empty, infinite and $\sup A_{p(x), q(x)}=+\infty$.

Remark 3.9 When $p$ and $q$ are constants, the equation (11) reduces to

$$
-\operatorname{div}\left(\frac{|\nabla u|^{p-2} \nabla u}{\|\nabla u\|_{p}^{p-1}}\right)=\Lambda\left(\frac{\sqrt[p]{p}}{\sqrt[q]{q}}\right) \frac{|u|^{q-2} u}{\|u\|_{q}^{q-1}}
$$

and the first eigenvalue is given by

$$
\Lambda_{1}=\frac{\sqrt[q]{q}}{\sqrt[p]{p}} \inf _{W_{0}^{1, p}(\Omega) \backslash\{0\}} \frac{\|\nabla v\|_{p}}{\|v\|_{q}} .
$$

\section{Extremal functions for $\frac{\|\cdot\|_{l p(x)}}{\|\cdot\|_{\infty}}$}

We recall the Morrey inequality, valid for $p>N$ :

$$
\|u\|_{C^{0, \gamma}(\bar{\Omega})} \leq C\|\nabla u\|_{L^{p}(\Omega)}, \quad \forall u \in W_{0}^{1, p}(\Omega),
$$

where $\gamma:=1-\frac{N}{p}$ and the positive constant $C$ depends only on $N, \Omega$ and $p$. An important consequence of this inequality is the compactness of the embedding

$$
W_{0}^{1, p}(\Omega) \hookrightarrow C(\bar{\Omega}) .
$$

Combining this fact with Proposition 2.4, we can verify the compactness of the embeddings

$$
W_{0}^{1, l p(x)}(\Omega) \hookrightarrow C(\bar{\Omega}) \quad \text { and } \quad W_{0}^{1, l p(x)}(\Omega) \hookrightarrow L^{j q(x)}(\Omega),
$$

where here, and throughout this section: 
- $l, j \in \mathbb{N}$, with $l \geq N$;

- $p \in C^{1}(\bar{\Omega})$, with $1<p^{-} \leq p^{+}<\infty$ (so that $\left.l p(x) \geq l p^{-}>N\right)$;

- $q \in C(\bar{\Omega})$ with $1<q^{-} \leq q^{+}<\infty$.

The following lemma is proved in [18].

Lemma 4.1 If $u \in L^{\infty}(\Omega)$, then

$$
\lim _{j \rightarrow \infty}\|u\|_{j q(x)}=\|u\|_{\infty}
$$

The previous lemma is also valid if we consider an increasing sequence of functions $\left(q_{j}\right) \subset C(\bar{\Omega})$ such that $q_{j} \rightarrow \infty$ uniformly.

Let us define

$$
\Lambda_{l, j}:=\inf \left\{\frac{\|\nabla v\|_{l p(x)}}{\|v\|_{j q(x)}}: v \in W_{0}^{1, l p(x)}(\Omega) \backslash\{0\}\right\}
$$

and

$$
\mu_{l}:=\inf \left\{\frac{\|\nabla v\|_{l p(x)}}{\|v\|_{\infty}}: v \in W_{0}^{1, l p(x)}(\Omega) \backslash\{0\}\right\} .
$$

Proposition 4.2 One has,

$$
\lim _{j \rightarrow \infty} \Lambda_{l, j}=\mu_{l}
$$

Proof. It follows from Lemma 4.1 that

$$
\limsup _{j \rightarrow \infty} \Lambda_{l, j} \leq \lim _{j \rightarrow \infty} \frac{\|\nabla v\|_{l p(x)}}{\|v\|_{j q(x)}}=\frac{\|\nabla v\|_{l p(x)}}{\|v\|_{\infty}}, \quad \forall v \in W_{0}^{1, l p(x)}(\Omega) \backslash\{0\} .
$$

Therefore,

$$
\limsup _{j \rightarrow \infty} \Lambda_{l, j} \leq \mu_{l}
$$

For any $j \geq 1$, let $u_{l, j}$ denote the extremal of $\Lambda_{l, j}$, that is,

$$
\Lambda_{l, j}=\frac{\left\|\nabla u_{l, j}\right\|_{l p(x)}}{\left\|u_{l, j}\right\|_{j q(x)}}
$$

It follows from (6) that

$$
\left\|u_{l, j}\right\|_{j q(x)} \leq|\Omega|^{\alpha_{j}}\left\|u_{l, j}\right\|_{\infty},
$$

where

$$
\alpha_{j}:=\left\{\begin{array}{lll}
\frac{1}{j q^{+}} & \text {if } & |\Omega| \leq 1 \\
\frac{1}{j q^{-}} & \text {if } & |\Omega|>1
\end{array}\right.
$$


Hence,

$$
\frac{\mu_{l}}{|\Omega|^{\alpha_{j}}} \leq \frac{\left\|\nabla u_{l, j}\right\|_{l p(x)}}{|\Omega|^{\alpha_{j}}\left\|u_{l, j}\right\|_{\infty}} \leq \frac{\left\|\nabla u_{l, j}\right\|_{l p(x)}}{\left\|u_{l, j}\right\|_{j q(x)}}=\Lambda_{l, j}
$$

and by making $j \rightarrow \infty$ we obtain

$$
\mu_{l} \leq \liminf _{j \rightarrow \infty} \Lambda_{l, j}
$$

concluding thus the proof of (19).

We say that $u \in W_{0}^{1, l p(x)}(\Omega)$ is an extremal function of $\mu_{l}$ if

$$
\mu_{l}=\frac{\|\nabla u\|_{l p(x)}}{\|u\|_{\infty}}
$$

Proposition 4.3 Let $l \geq N$ be fixed. There exists $j_{m} \rightarrow \infty$ and a function $w_{l} \in W_{0}^{1, l p(x)}(\Omega) \cap$ $C(\bar{\Omega})$ such that $u_{l, j_{m}} \rightarrow w_{l}$ strongly in $C(\bar{\Omega})$ and also in $W_{0}^{1, l p(x)}(\Omega)$. Moreover, $w_{l}$ is an extremal function of $\mu_{l}$.

Proof. Let $u_{l, j}$ denote the extremal function of $\Lambda_{l, j}$. Without loss of generality we assume that $\left\|u_{l, j}\right\|_{j q(x)}=1$. Since the sequence $\left(u_{l, j}\right)_{j \geq 1}$ is uniformly bounded in $W_{0}^{1, l p(x)}(\Omega)$, there exist $j_{m} \rightarrow \infty$ and $w_{l} \in W_{0}^{1, l p(x)}(\Omega) \subset C(\bar{\Omega})$ such that $u_{l, j_{m}}$ converges to $w_{l}$, weakly in $W_{0}^{1, l p(x)}(\Omega)$ and strongly in $C(\bar{\Omega})$. It follows from (20) that

$$
1=\lim _{m \rightarrow \infty}\left\|u_{l, j_{m}}\right\|_{j q(x)} \leq \lim _{m \rightarrow \infty}\left\|u_{l, j_{m}}\right\|_{\infty}=\left\|w_{l}\right\|_{\infty},
$$

so that

$$
\mu_{l} \leq \frac{\left\|\nabla w_{l}\right\|_{l p(x)}}{\left\|w_{l}\right\|_{\infty}} \leq\left\|\nabla w_{l}\right\|_{l p(x)} \leq \lim _{m \rightarrow \infty}\left\|\nabla u_{l, j_{m}}\right\|_{l p(x)}=\lim _{m \rightarrow \infty} \Lambda_{l, j_{m}}=\mu_{l}
$$

Hence,

$$
\left\|w_{l}\right\|_{\infty}=1, \quad \mu_{l}=\left\|\nabla w_{l}\right\|_{l p(x)}=\lim _{m \rightarrow \infty}\left\|\nabla u_{l, j_{m}}\right\|_{l p(x)},
$$

implying that $w_{l}$ is an extremal function of $\mu_{l}$ and that $u_{l, j_{m}} \rightarrow w_{l}$ strongly in $W_{0}^{1, l p(x)}(\Omega)$.

Now, by adapting arguments of [19] we characterize of the extremal functions of $\mu_{l}$. For this, let us denote by $\Gamma_{u}$ the set of the points where a function $u \in C(\bar{\Omega})$ assumes its uniform norm, that is

$$
\Gamma_{u}:=\left\{x \in \bar{\Omega}:|u(x)|=\|u\|_{\infty}\right\}
$$

Lemma 4.4 Let $u, \eta \in C(\bar{\Omega})$, with $u \neq \equiv$. One has

$$
\lim _{\epsilon \rightarrow 0^{+}} \frac{\|u+\epsilon \eta\|_{\infty}-\|u\|_{\infty}}{\epsilon}=\max \left\{\operatorname{sgn}(u(x)) \eta(x): x \in \Gamma_{u}\right\} .
$$


Proof. Let $r>1, \delta>0$ and $t \in \mathbb{R}$. Since the function $s \mapsto|s|^{r-2} s$ is increasing we have

$$
\frac{|t+\delta|^{r}}{r}=\int_{0}^{t+\delta}|s|^{r-2} s \mathrm{~d} s=\frac{|t|^{r}}{r}+\int_{t}^{t+\delta}|s|^{r-2} s \mathrm{~d} s \geq \frac{|t|^{r}}{r}+|t|^{r-2} t \delta .
$$

Thus, for $x_{0} \in \Gamma_{u}, \eta \in C(\bar{\Omega})$ and $\epsilon>0$, we obtain

$$
\frac{\|u+\epsilon \eta\|_{\infty}^{r}}{r} \geq \frac{\left|u\left(x_{0}\right)+\epsilon \eta\left(x_{0}\right)\right|^{r}}{r} \geq \frac{\left|u\left(x_{0}\right)\right|^{r}}{r}+\left|u\left(x_{0}\right)\right|^{r-2} u\left(x_{0}\right) \epsilon \eta\left(x_{0}\right) .
$$

Making $r \rightarrow 1^{+}$(and using that $\left|u\left(x_{0}\right)\right|=\|u\|_{\infty} \neq 0$ ) we arrive at the inequality

$$
\frac{\|u+\epsilon \eta\|_{\infty}-\|u\|_{\infty}}{\epsilon} \geq \operatorname{sgn}\left(u\left(x_{0}\right)\right) \eta\left(x_{0}\right)
$$

which, in view of the arbitrariness of $x_{0} \in \Gamma_{u}$, implies that

$$
\liminf _{\epsilon \rightarrow 0^{+}} \frac{\|u+\epsilon \eta\|_{\infty}-\|u\|_{\infty}}{\epsilon} \geq \max \left\{\operatorname{sgn}(u(x)) \eta(x): x \in \Gamma_{u}\right\}
$$

In order to conclude this proof we will obtain the reverse inequality for $\lim \sup _{\epsilon \rightarrow 0^{+}}$. For this, we take $\epsilon_{m} \rightarrow 0^{+}$such that

$$
\limsup _{\epsilon \rightarrow 0^{+}} \frac{\|u+\epsilon \eta\|_{\infty}-\|u\|_{\infty}}{\epsilon}=\limsup _{m \rightarrow \infty} \frac{\left\|u+\epsilon_{m} \eta\right\|_{\infty}-\|u\|_{\infty}}{\epsilon_{m}}
$$

and select a sequence $\left(x_{m}\right) \subset \bar{\Omega}$ satisfying

$$
\left|u\left(x_{m}\right)+\epsilon_{m} \eta\left(x_{m}\right)\right|=\left\|u+\epsilon_{m} \eta\right\|_{\infty} .
$$

We can assume (by passing to a subsequence, if necessary) that $x_{m} \rightarrow x_{0} \in \bar{\Omega}$. Of course, $x_{0} \in \Gamma_{u}$ since $u+\epsilon_{m} \eta \rightarrow u$ in $C(\bar{\Omega})$.

Since $\|u\|_{\infty} \geq\left|u\left(x_{m}\right)\right|$ we have

$$
\limsup _{m \rightarrow \infty} \frac{\left\|u+\epsilon_{m} \eta\right\|_{\infty}-\|u\|_{\infty}}{\epsilon_{m}} \leq \limsup _{m \rightarrow \infty} \frac{\left|u\left(x_{m}\right)+\epsilon_{m} \eta\left(x_{m}\right)\right|-\left|u\left(x_{m}\right)\right|}{\epsilon_{m}}
$$

and since $u\left(x_{m}\right)+\epsilon_{m} \eta\left(x_{m}\right) \rightarrow u\left(x_{0}\right)$ we have, for all $m$ large enough,

$$
\frac{\left|u\left(x_{m}\right)+\epsilon_{m} \eta\left(x_{m}\right)\right|-\left|u\left(x_{m}\right)\right|}{\epsilon_{m}}=\left\{\begin{array}{cc}
\eta\left(x_{m}\right), & u\left(x_{0}\right)>0 \\
-\eta\left(x_{m}\right), & u\left(x_{0}\right)<0
\end{array}=\operatorname{sgn}\left(u\left(x_{0}\right)\right) \eta\left(x_{m}\right) .\right.
$$

It follows that

$$
\limsup _{\epsilon \rightarrow 0^{+}} \frac{\|u+\epsilon \eta\|_{\infty}-\|u\|_{\infty}}{\epsilon} \leq \operatorname{sgn}\left(u\left(x_{0}\right)\right) \eta\left(x_{0}\right) \leq \max \left\{\operatorname{sgn}(u(x)) \eta(x): x \in \Gamma_{u}\right\} .
$$


Theorem 4.5 Let $l \geq N$ be fixed. A function $u \in W_{0}^{1, l p(x)}(\Omega) \backslash\{0\}$ is extremal of $\mu_{l}$ if, and only if, $\Gamma_{u}=\left\{x_{0}\right\}$ for some $x_{0} \in \Omega$ and

$$
\int_{\Omega}\left|\frac{\nabla u}{K_{l}(u)}\right|^{l p(x)-2} \frac{\nabla u}{K_{l}(u)} \cdot \nabla \eta \mathrm{d} x=\mu_{l} S_{l}(u) \operatorname{sgn}\left(u\left(x_{0}\right)\right) \eta\left(x_{0}\right), \quad \forall \eta \in W_{0}^{1, l p(x)}(\Omega),
$$

where

$$
K_{l}(u):=\|\nabla u\|_{l p(x)} \quad \text { and } \quad S_{l}(u):=\int_{\Omega}\left|\frac{\nabla u}{K_{l}(u)}\right|^{l p(x)} \mathrm{d} x .
$$

Proof. Let $u \in W_{0}^{1, l p(x)}(\Omega) \backslash\{0\}$ be an extremal function of $\mu_{l}$ and fix $\eta \in W_{0}^{1, l p(x)}(\Omega)$. Then

$$
\mu_{l}=\frac{\|\nabla u\|_{l p(x)}}{\|u\|_{\infty}} \leq \frac{\|\nabla u+\epsilon \nabla \eta\|_{l p(x)}}{\|u+\epsilon \eta\|_{\infty}}, \quad \forall \epsilon>0
$$

It follows that

$$
\begin{aligned}
0 & \leq \lim _{\epsilon \rightarrow 0^{+}} \frac{1}{\epsilon}\left(\frac{\|\nabla u+\epsilon \nabla \eta\|_{l p(x)}}{\|u+\epsilon \eta\|_{\infty}}-\frac{\|\nabla u\|_{l p(x)}}{\|u\|_{\infty}}\right) \\
& =\lim _{\epsilon \rightarrow 0^{+}} \frac{1}{\epsilon}\left(\frac{\|\nabla u+\epsilon \nabla \eta\|_{l p(x)}}{\|u+\epsilon \eta\|_{\infty}}-\frac{\|\nabla u\|_{l p(x)}}{\|u+\epsilon \eta\|_{\infty}}+\frac{\|\nabla u\|_{l p(x)}}{\|u+\epsilon \eta\|_{\infty}}-\frac{\|\nabla u\|_{l p(x)}}{\|u\|_{\infty}}\right) \\
& =\frac{1}{\|u\|_{\infty}}\left(\frac{1}{S_{l}(u)} \int_{\Omega}\left|\frac{\nabla u}{K_{l}(u)}\right|^{l p(x)-2} \frac{\nabla u}{K_{l}(u)} \cdot \nabla \eta \mathrm{d} x-\frac{\|\nabla u\|_{l p(x)}}{\|u\|_{\infty}} \max \left\{\operatorname{sgn}(u(x)) \eta(x): x \in \Gamma_{u}\right\}\right) \\
& =\frac{1}{\|u\|_{\infty}}\left(\frac{1}{S_{l}(u)} \int_{\Omega}\left|\frac{\nabla u}{K_{l}(u)}\right|^{l p(x)-2} \frac{\nabla u}{K_{l}(u)} \cdot \nabla \eta \mathrm{d} x-\mu_{l} \max \left\{\operatorname{sgn}(u(x)) \eta(x): x \in \Gamma_{u}\right\}\right),
\end{aligned}
$$

where we have used Lemma 3.2 and Lemma 4.4 .

Therefore,

$$
\int_{\Omega}\left|\frac{\nabla u}{K_{l}(u)}\right|^{l p(x)-2} \frac{\nabla u}{K_{l}(u)} \cdot \nabla \eta \mathrm{d} x \geq \mu_{l} S_{l}(u) \max \left\{\operatorname{sgn}(u(x)) \eta(x): x \in \Gamma_{u}\right\} .
$$

Now, by replacing $\eta$ by $-\eta$ in this inequality we obtain

$$
\int_{\Omega}\left|\frac{\nabla u}{K_{l}(u)}\right|^{l p(x)-2} \frac{\nabla u}{K_{l}(u)} \cdot \nabla \eta \mathrm{d} x \leq \mu_{l} S_{l}(u) \min \left\{\operatorname{sgn}(u(x)) \eta(x): x \in \Gamma_{u}\right\} .
$$

We then conclude from (22) and (23) that

$$
\begin{aligned}
\mu_{l} S_{l}(u) \min \left\{\operatorname{sgn}(u(x)) \eta(x): x \in \Gamma_{u}\right\} & =\int_{\Omega}\left|\frac{\nabla u}{K_{l}(u)}\right|^{l p(x)-2} \frac{\nabla u}{K_{l}(u)} \cdot \nabla \eta \mathrm{d} x \\
& =\mu_{l} S_{l}(u) \max \left\{\operatorname{sgn}(u(x)) \eta(x): x \in \Gamma_{u}\right\} .
\end{aligned}
$$


Taking into account the arbitrariness of $\eta \in W_{0}^{1, l p(x)}(\Omega)$ this implies that $\Gamma_{u}=\left\{x_{0}\right\}$ for some $x_{0} \in \Omega$. Consequently, $u$ satisfies (21) for $x_{0}$.

Reciprocally, if $u \in W_{0}^{1, l p(x)}(\Omega) \backslash\{0\}$ is such that $\Gamma_{u}=\left\{x_{0}\right\}$ for some $x_{0} \in \Omega$ and, additionally, satisfies (21) for this point, we can choose $\eta=u$ in (21) to get

$$
\int_{\Omega}\left|\frac{\nabla u}{K_{l}(u)}\right|^{l p(x)-2} \frac{\nabla u}{K_{l}(u)} \cdot \nabla u \mathrm{~d} x=\mu_{l} S_{l}(u) \operatorname{sgn}\left(u\left(x_{0}\right)\right) u\left(x_{0}\right)=\mu_{l} S_{l}(u)\|u\|_{\infty},
$$

so that

$$
\mu_{l}=\frac{\|\nabla u\|_{l p(x)}}{\|u\|_{\infty}}
$$

Corollary 4.6 Extremal functions of $\mu_{l}$ do not change sign in $\Omega$.

Proof. Let $u \in W_{0}^{1, l p(x)}(\Omega) \backslash\{0\}$ be an extremal function of $\mu_{l}$ and $x_{0} \in \Omega$ the only point where $u$ achieves its uniform norm. If $u\left(x_{0}\right)>0$, Theorem 4.5 yields

$$
\int_{\Omega}\left|\frac{\nabla u}{K_{l}(u)}\right|^{l p(x)-2} \frac{\nabla u}{K_{l}(u)} \cdot \nabla \eta \mathrm{d} x=\mu_{l} S_{l}(u) \eta\left(x_{0}\right) \geq 0,
$$

for all nonnegative $\eta \in W_{0}^{1, l p(x)}(\Omega)$. Proposition 2.8 then implies that $u>0$ in $\Omega$. If $u\left(x_{0}\right)<0$ we repeat the argument for the extremal function $-u$.

We can say that

$$
-\operatorname{div}\left(\left|\frac{\nabla u}{K_{l}(u)}\right|^{l p(x)-2} \frac{\nabla u}{K_{l}(u)}\right)=\mu_{l} S_{l}(u) \operatorname{sgn}\left(u\left(x_{0}\right)\right) \delta_{x_{0}},
$$

is the Euler-Lagrange equation associated with the minimization problem defined by (18), where $\delta_{x_{0}}$ is the Dirac delta function concentrated in $x_{0}$. We recall that $\delta_{x_{0}}$ is defined by

$$
\delta_{x_{0}}(\eta)=\eta\left(x_{0}\right), \quad \forall \eta \in W_{0}^{1, l p(x)}(\Omega) .
$$

Thus, the extremal functions of $\mu_{l}$ are precisely the weak solutions of (24) in the sense of (21).

Remark 4.7 Consider a function $v \in W_{0}^{1, l p(x)}(\Omega)$ such that $\left|v\left(x_{0}\right)\right|=\|v\|_{\infty}$ for some $x_{0} \in \Omega$ and suppose that this function satisfies the equation

$$
\int_{\Omega}\left|\frac{\nabla v}{K_{l}(v)}\right|^{l p(x)-2} \frac{\nabla v}{K_{l}(v)} \cdot \nabla \eta \mathrm{d} x=\mu\left(\int_{\Omega}\left|\frac{\nabla v}{K_{l}(v)}\right|^{l p(x)} \mathrm{d} x\right) \operatorname{sgn}\left(v\left(x_{0}\right)\right) \eta\left(x_{0}\right), \quad \forall \eta \in W_{0}^{1, l p(x)}(\Omega)
$$

where $\mu \in \mathbb{R}$. By making $\eta=v$, it follows that

$$
\mu=\frac{K_{l}(v)}{\left|v\left(x_{0}\right)\right|}=\frac{\|\nabla v\|_{l p(x)}}{\|v\|_{\infty}} \geq \mu_{l}
$$


Thus, $\mu_{l}$ can be interpreted as the first eigenvalue of (21). Moreover, for a given natural $j \geq 1$, we know, from Section 3, that there exists a sequence

$$
0<\lambda_{1}^{l, j} \leq \lambda_{2}^{l, j} \leq \cdots \leq \lambda_{n}^{l, j} \leq \lambda_{n+1}^{l, j} \leq \cdots
$$

of eigenvalues, where the exponent functions, in this case, are $l p(x)$ and $j q(x)$. Proposition 4.2 then says that

$$
\lim _{j \rightarrow \infty} \lambda_{1}^{l, j}=\mu_{l}
$$

\section{The limit problem as $l \rightarrow \infty$}

In this section we maintain $p \in C^{1}(\bar{\Omega})$, with $1<p^{-} \leq p^{+}<\infty$. For each natural $l \geq N$ we denote by $w_{l}$ a positive, $L^{\infty}$-normalized extremal function of $\mu_{l}$. Thus,

$$
w_{l} \in W_{0}^{1, l p(x)}(\Omega), \quad\left\|w_{l}\right\|_{\infty}=1, \quad w_{l}>0 \quad \text { in } \Omega,
$$

and

$$
\mu_{l}=\left\|\nabla w_{l}\right\|_{l p(x)} \leq \frac{\|\nabla v\|_{l p(x)}}{\|v\|_{\infty}}, \quad \forall v \in W_{0}^{1, l p(x)}(\Omega) \backslash\{0\} .
$$

We will also denote by $x_{0}^{l}$ the only maximum point of $w_{l}$. According to the previous section, $w_{l}$ satisfies

$$
-\Delta_{l p(x)}\left(\frac{w_{l}}{K_{l}\left(w_{l}\right)}\right)=\mu_{l} S_{l}\left(w_{l}\right) \delta_{x_{0}^{l}} \quad \text { in } \quad \Omega,
$$

where $\delta_{x_{0}^{l}}$ is the Dirac delta function concentrated in $x_{0}^{l}$,

$$
K_{l}\left(w_{l}\right)=\left\|\nabla w_{l}\right\|_{l p(x)}=\mu_{l} \quad \text { and } \quad S_{l}\left(w_{l}\right):=\int_{\Omega}\left|\frac{\nabla w_{l}}{K_{l}\left(w_{l}\right)}\right|^{l p(x)} \mathrm{d} x .
$$

Hence,

$$
\int_{\Omega}\left|\frac{\nabla w_{l}}{K_{l}\left(w_{l}\right)}\right|^{l p(x)-2} \frac{\nabla w_{l}}{K_{l}\left(w_{l}\right)} \cdot \nabla \eta \mathrm{d} x=\mu_{l} S_{l}\left(w_{l}\right) \eta\left(x_{0}^{l}\right), \quad \forall \eta \in W_{0}^{1, l p(x)}(\Omega) .
$$

Let us define

$$
\Lambda_{\infty}:=\inf \left\{\frac{\|\nabla v\|_{\infty}}{\|v\|_{\infty}}: v \in W_{0}^{1, \infty}(\Omega) \backslash\{0\}\right\} .
$$

It is a well-known fact that

$$
\Lambda_{\infty}=\frac{\|\nabla d\|_{\infty}}{\|d\|_{\infty}}=\frac{1}{\|d\|_{\infty}}
$$

where $d$ denotes the distance function to the boundary $\partial \Omega$, defined by

$$
d(x):=\inf _{y \in \partial \Omega}|x-y|, \quad x \in \bar{\Omega} .
$$

We recall that

$$
d \in W_{0}^{1, \infty}(\Omega) \quad \text { and } \quad|\nabla d|=1 \text { a.e. in } \Omega \text {. }
$$


Lemma 5.1 Let $\alpha:=\int_{\Omega} \frac{\mathrm{d} x}{p(x)}$ and $e:=\exp (1)$. If $\alpha e<m<l$, then

$$
\|u\|_{m p(x)} \leq\|u\|_{l p(x)} \quad \forall u \in L^{l p(x)}(\Omega) .
$$

Proof. When $u \equiv 0$ the equality holds trivially in the above inequality. Thus, we fix $u \in$ $L^{l p(x)}(\Omega) \backslash\{0\}$ and denote the modular functions associated to $L^{m p(x)}(\Omega)$ and $L^{l p(x)}(\Omega)$ by $\rho_{m}$ and $\rho_{l}$, respectively.

By Hölder's inequality

$$
\begin{aligned}
\rho_{m}(u) & =\int_{\Omega}|u|^{m p(x)} \frac{\mathrm{d} x}{m p(x)} \\
& =\frac{1}{m} \int_{\Omega} \frac{|u|^{m p(x)}}{p(x)^{m / l}}\left(\frac{1}{p(x)}\right)^{\frac{l-m}{l}} \mathrm{~d} x \\
& \leq \frac{1}{m}\left(\int_{\Omega} \frac{|u|^{l p(x)}}{p(x)}\right)^{\frac{m}{l}}\left(\int_{\Omega} \frac{\mathrm{d} x}{p(x)}\right)^{1-\frac{m}{l}}=\frac{1}{m}\left(l \rho_{l}(u)\right)^{\frac{m}{l}} \alpha^{1-\frac{m}{l}}=\left(\frac{f(l)}{f(m)} \rho_{l}(u)^{\frac{1}{l}}\right)^{m},
\end{aligned}
$$

where $f(s)=\left(\frac{s}{\alpha}\right)^{\frac{1}{s}}$. Since $f$ is decreasing in $(\alpha e, \infty)$ and $\alpha e<m<l$ we have

$$
\rho_{m}(u)^{\frac{1}{m}} \leq \frac{f(l)}{f(m)} \rho_{l}(u)^{\frac{1}{l}} \leq \rho_{l}(u)^{\frac{1}{l}} .
$$

Hence, by taking $a=\|u\|_{l p(x)} \neq 0$ and applying item b) of Proposition 2.2 we conclude that

$$
\rho_{m}\left(\frac{u}{a}\right)^{\frac{1}{m}} \leq \rho_{l}\left(\frac{u}{a}\right)^{\frac{1}{l}}=1
$$

and then that $\left\|\frac{u}{a}\right\|_{m p(x)} \leq 1$. This implies that

$$
\|u\|_{m p(x)} \leq a=\|u\|_{l p(x)} .
$$

Proposition 5.2 There exists a subsequence of $\left(w_{l}\right)_{l \in \mathbb{N}}$ converging strongly in $C(\bar{\Omega})$ to a nonnegative function $w_{\infty} \in W_{0}^{1, \infty}(\Omega) \backslash\{0\}$ such that

$$
\lim _{l \rightarrow \infty} \mu_{l}=\Lambda_{\infty}=\left\|\nabla w_{\infty}\right\|_{\infty} .
$$

Moreover,

$$
0 \leq w_{\infty}(x) \leq \frac{d(x)}{\|d\|_{\infty}}, \quad \text { for almost every } x \in \Omega
$$

Proof. Since

$$
\left\|\nabla w_{l}\right\|_{l p(x)}=\mu_{l} \leq \frac{\|\nabla d\|_{l p(x)}}{\|d\|_{\infty}}
$$


we can apply Lemma 4.1 to get

$$
\limsup _{l \rightarrow \infty} \mu_{l} \leq \frac{\|\nabla d\|_{\infty}}{\|d\|_{\infty}}=\Lambda_{\infty} .
$$

Let us take a natural $m>\alpha e$, where $\alpha$ is given by Lemma 5.1, and a subsequence $\left(\mu_{l_{n}}\right)_{n \in \mathbb{N}}$ such that

$$
\lim _{n \rightarrow \infty} \mu_{l_{n}}=\liminf _{l \rightarrow \infty} \mu_{l} .
$$

Combining Lemma 5.1 with (27) we conclude that the sequence $\left(w_{l_{n}}\right)_{l_{n}>m}$ is bounded in $W_{0}^{1, m p(x)}(\Omega)$, since

$$
\limsup _{n \rightarrow \infty}\left\|\nabla w_{l_{n}}\right\|_{m p(x)} \leq \limsup _{n \rightarrow \infty}\left\|\nabla w_{l_{n}}\right\|_{l_{n} p(x)} \leq \Lambda_{\infty}
$$

Thus, up to a subsequence, we can assume that there exists $w_{\infty} \in W_{0}^{1, m p(x)}(\Omega)$, such that $w_{l_{n}}$ converges to $w_{\infty}$, weakly in $W_{0}^{1, m p(x)}(\Omega)$ and uniformly in $\bar{\Omega}$.

The uniform convergence, implies that $\left\|w_{\infty}\right\|_{\infty}=1$ (since $\left\|w_{l_{n}}\right\|_{\infty}=1$ ). The weak convergence in $W_{0}^{1, m p(x)}(\Omega)$ implies that

$$
\left\|\nabla w_{\infty}\right\|_{m p(x)} \leq \liminf _{n \rightarrow \infty}\left\|\nabla w_{l_{n}}\right\|_{m p(x)} .
$$

Now, applying Lemma 5.1 again, we conclude that

$$
\liminf _{n \rightarrow \infty}\left\|\nabla w_{l_{n}}\right\|_{m p(x)} \leq \liminf _{n \rightarrow \infty}\left\|\nabla w_{l_{n}}\right\|_{l_{n} p(x)}=\lim _{n \rightarrow \infty} \mu_{l_{n}}=\liminf _{l \rightarrow \infty} \mu_{l} .
$$

Hence, (29) yields

$$
\left\|\nabla w_{\infty}\right\|_{m p(x)} \leq \liminf _{l \rightarrow \infty} \mu_{l}
$$

Repeating the above arguments we conclude that $w_{\infty}$ is the weak limit of a subsequence of $\left(w_{l_{n}}\right)_{n \in \mathbb{N}}$ in $W_{0}^{1, s p(x)}(\Omega)$, for any $s>m$. This fact implies that $w_{\infty} \in W_{0}^{1, \infty}(\Omega)$. Then, by making $m \rightarrow \infty$ in (30), using Lemma 4.1 and (28) we conclude that

$$
\Lambda_{\infty} \leq\left\|\nabla w_{\infty}\right\|_{\infty} \leq \liminf _{l \rightarrow \infty} \mu_{l} \leq \limsup _{l \rightarrow \infty} \mu_{l} \leq \Lambda_{\infty}
$$

which gives (25).

Since the Lipschitz constant of $w_{\infty}$ is $\left\|\nabla w_{\infty}\right\|_{\infty}=\Lambda_{\infty}=\frac{1}{\|d\|_{\infty}}$, we have

$$
\|d\|_{\infty}\left|w_{\infty}(x)-w_{\infty}(y)\right| \leq|x-y|
$$

for almost all $x \in \Omega$ and $y \in \partial \Omega$. Since $w \equiv 0$ on the boundary $\partial \Omega$, we obtain

$$
\|d\|_{\infty} w_{\infty}(x) \leq \inf _{y \in \partial \Omega}|x-y|=d(x) .
$$

We show in the sequel that the functions $w_{\infty}$ and $d$ have a maximum point in common, which is obtained as a cluster point of the sequence $\left(x_{0}^{l}\right)_{l \in \mathbb{N}}$. 
Corollary 5.3 There exists $x_{\star} \in \Omega$ such that

$$
w_{\infty}\left(x_{\star}\right)=\left\|w_{\infty}\right\|_{\infty}=1 \quad \text { and } \quad d\left(x_{\star}\right)=\|d\|_{\infty}
$$

Proof. Let $\left(w_{l_{n}}\right)_{n \in \mathbb{N}}$ be a sequence converging uniformly to $w_{\infty}$, which is given by Proposition 5.2. Up to a subsequence, we can assume that $x_{0}^{l_{n}} \rightarrow x_{\star} \in \bar{\Omega}$. Since $w_{l_{n}}\left(x_{0}^{l_{n}}\right)=1=\left\|w_{l_{n}}\right\|_{\infty}$ we have $w_{\infty}\left(x_{\star}\right)=1=\left\|w_{\infty}\right\|_{\infty}$, showing that $x_{\star} \in \Omega$. The conclusion stems from (26), since

$$
1=w_{\infty}\left(x_{\star}\right) \leq \frac{d\left(x_{\star}\right)}{\|d\|_{\infty}} \leq 1 .
$$

In the sequel we recall the concept of viscosity solutions for an equation of the form

$$
H\left(x, u, \nabla u, D^{2} u\right)=0 \quad \text { in } D
$$

where $H$ is a partial differential operator of second order and $D$ denotes a bounded domain of $\mathbb{R}^{N}$.

Definition 5.4 Let $\phi \in C^{2}(D), x_{0} \in D$ and $u \in C(D)$. We say that $\phi$ touches $u$ from below at $x_{0}$ if

$$
\phi\left(x_{0}\right)=u\left(x_{0}\right) \quad \text { and } \quad \phi(x)<u(x), \quad x \neq x_{0}
$$

Analogously, we say that $\phi$ touches $u$ from above at $x_{0}$ if

$$
\phi\left(x_{0}\right)=u\left(x_{0}\right) \quad \text { and } \quad \phi(x)>u(x), \quad x \neq x_{0} .
$$

Definition 5.5 We say that $u \in C(D)$ is a viscosity supersolution of the equation (31) if, whenever $\phi \in C^{2}(D)$ touches $u$ from below at a point $x_{0} \in D$, we have

$$
H\left(x_{0}, \phi\left(x_{0}\right), \nabla \phi\left(x_{0}\right), D^{2} \phi\left(x_{0}\right)\right) \leq 0 .
$$

Analogously, we say that $u$ is a viscosity subsolution if, whenever $\psi \in C^{2}(D)$ touches $u$ from above at a point $x_{0} \in D$, we have

$$
H\left(x_{0}, \psi\left(x_{0}\right), \nabla \psi\left(x_{0}\right), D^{2} \psi\left(x_{0}\right)\right) \geq 0
$$

And we say that $u$ is a viscosity solution, if $u$ is both a viscosity supersolution and a viscosity subsolution.

Note that the differential operator $H$ is evaluated for the test functions only at the touching point.

In order to interpret the equation

$$
\Delta_{p(x)}\left(\frac{u}{K(u)}\right)=0 \text { in } \Omega
$$


in the viscosity sense, we need to find the expression of the corresponding differential operator $H$. If $\phi$ is a function of class $C^{2}$, one can verify that the $p(x)$-Laplacian is given by

$$
\Delta_{p(x)} \phi=|\nabla \phi|^{p(x)-4}\left\{|\nabla \phi|^{2} \Delta \phi+(p(x)-2) \Delta_{\infty} \phi+|\nabla \phi|^{2} \ln |\nabla \phi|\langle\nabla \phi, \nabla p\rangle\right\}
$$

where $\langle\nabla \phi, \nabla p\rangle=\nabla \phi \cdot \nabla p$ and $\Delta_{\infty}$ denotes the $\infty$-Laplacian defined by

$$
\Delta_{\infty} v:=\frac{1}{2}\left\langle\nabla v, \nabla|\nabla v|^{2}\right\rangle=\sum_{i, j=1}^{N} \frac{\partial v}{\partial x_{i}} \frac{\partial v}{\partial x_{j}} \frac{\partial^{2} v}{\partial x_{i} \partial x_{j}}
$$

Thus, for a positive constant $t$ one can check that

$$
\Delta_{p(x)}(t \phi)=t^{p(x)-1}|\nabla \phi|^{p(x)-4}\left\{|\nabla \phi|^{2} \Delta \phi+(p(x)-2) \Delta_{\infty} \phi+|\nabla \phi|^{2} \ln (|\nabla(t \phi)|)\langle\nabla \phi, \nabla p\rangle\right\}
$$

and by choosing $t=K(u)^{-1}$ we obtain from (33) that the equation (32) can be rewritten in the form (31) with

$$
H\left(x, u, \nabla u, D^{2} u\right):=|\nabla u|^{p(x)-4}\left\{|\nabla u|^{2} \Delta \phi+(p(x)-2) \Delta_{\infty} u+|\nabla u|^{2} \ln \left(\frac{|\nabla u|}{K(u)}\right)\langle\nabla u, \nabla p\rangle\right\},
$$

where we are assuming that $p(x) \geq 2$.

Proposition 5.6 If $u \in C(\Omega) \cap W_{0}^{1, p(x)}(\Omega)$ is a weak solution of (32) with $K(u)=\|\nabla u\|_{p(x)}$, then $u$ is a viscosity solution of this equation.

Proof. We must prove that $u$ is both a viscosity supersolution and a viscosity subsolution of the equation

$$
H\left(x, u, \nabla u, D^{2} u\right)=0 \quad \text { in } \Omega
$$

for the differential operator $H$ defined by (34) with $K(u)=\|\nabla u\|_{p(x)}$.

By hypothesis, $u$ satisfies

$$
\int_{D}\left|\frac{\nabla u}{K(u)}\right|^{p(x)-2} \frac{\nabla u}{K(u)} \cdot \nabla \eta \mathrm{d} x=0, \quad \forall \eta \in W_{0}^{1, p(x)}(\Omega) .
$$

Let us prove by contradiction that $u$ is a viscosity supersolution. Thus, we suppose that there exist $x_{0} \in \Omega$ and $\phi \in C^{2}(\Omega)$ with $\phi$ touching $u$ from below at $x_{0}$ and satisfying

$$
H\left(x_{0}, \phi\left(x_{0}\right), \nabla \phi\left(x_{0}\right), D^{2} \phi\left(x_{0}\right)\right)>0 .
$$

By continuity, there exists a ball $B\left(x_{0}, r\right) \subset \Omega$, with $r$ small enough, such that

$$
H\left(x, \phi(x), \nabla \phi(x), D^{2} \phi(x)\right)>0, \quad \forall x \in B\left(x_{0}, r\right) .
$$

This means that

$$
\Delta_{p(x)}\left(\frac{\phi}{K(u)}\right)>0 \quad \text { in } B\left(x_{0}, r\right)
$$


Let $\varphi:=\phi+\frac{m}{2}$, with $m=\min _{\partial B\left(x_{0}, r\right)}(u-\phi)>0$, and take

$$
\eta=(\varphi-u)_{+} \chi_{B\left(x_{0}, r\right)} \in W_{0}^{1, p(x)}(\Omega) .
$$

We have $\varphi<u$ on $\partial B\left(x_{0}, r\right)$ and $\varphi\left(x_{0}\right)>u\left(x_{0}\right)$.

If $\eta \not \equiv 0$, we multiply (36) by $\eta$ and integrate by parts to obtain

$$
\int_{D}\left|\frac{\nabla \phi}{K(u)}\right|^{p(x)-2} \frac{\nabla \phi}{K(u)} \cdot \nabla \eta \mathrm{d} x<0 .
$$

Note that $\nabla \eta=\nabla \varphi-\nabla u=\nabla \phi-\nabla u$ in the set $\{\varphi>u\}$ (and $\nabla \eta=0$ in $B\left(x_{0}, r\right) \cap\{\varphi \leq u\}$ ). Thus, subtracting (35) from the above inequality we obtain

$$
\int_{\{\varphi>u\}}\left\langle\left|\frac{\nabla \phi}{K(u)}\right|^{p(x)-2} \frac{\nabla \phi}{K(u)}-\left|\frac{\nabla u}{K(u)}\right|^{p(x)-2} \frac{\nabla u}{K(u)}, \nabla \eta\right\rangle \mathrm{d} x<0
$$

implying thus that

$$
\int_{\{\varphi>u\}}\left\langle\left|\frac{\nabla \phi}{K(u)}\right|^{p(x)-2} \frac{\nabla \phi}{K(u)}-\left|\frac{\nabla u}{K(u)}\right|^{p(x)-2} \frac{\nabla u}{K(u)}, \frac{\nabla \phi}{K(u)}-\frac{\nabla u}{K(u)}\right\rangle \mathrm{d} x<0
$$

where the domain of integration is contained in $B\left(x_{0}, r\right)$. However, it is well known that

$$
\left\langle|Y|^{p-2} Y-|X|^{p-2} X, Y-X\right\rangle \geq 0 \quad \forall X, Y \in \mathbb{R}^{N} \text { and } p>1 .
$$

Thus, by making $Y=\frac{\nabla \phi}{K(u)}, X=\frac{\nabla u}{K(u)}$ and $p=p(x)$ we see that (37) cannot occurs. Therefore, $\eta \equiv 0$. But this implies that $\varphi \leq u$ in $B\left(x_{0}, r\right)$, contradicting $\varphi\left(x_{0}\right)>u\left(x_{0}\right)$.

Analogously, we can show that $u$ is a viscosity subsolution.

Our next result states that the function $w_{\infty}$ given by Proposition 5.2 is a viscosity solution of the equation

$$
\Delta_{\infty(x)}\left(\frac{u}{\|\nabla u\|_{\infty}}\right)=0
$$

in the punctured domain $D=\Omega \backslash\left\{x_{\star}\right\}$, where $x_{\star}$ is the maximum point of $w_{\infty}$ given by Corollary 5.3 and $\Delta_{\infty(x)}$ is the differential operator

$$
\Delta_{\infty(x)} u:=\Delta_{\infty} u+|\nabla u|^{2} \ln |\nabla u|\langle\nabla u, \nabla \ln p\rangle .
$$

Note that if $t>0$, then

$$
\Delta_{\infty(x)}\left(\frac{u}{t}\right):=t^{-3}\left\{\Delta_{\infty} u+|\nabla u|^{2} \ln \left|\nabla\left(\frac{u}{t}\right)\right|\langle\nabla u, \nabla \ln p\rangle\right\} .
$$

Lemma 5.7 ([22]) Suppose that $f_{n} \rightarrow f$ uniformly in $\bar{D}$, where $f_{n}, f \in C(\bar{D})$. If $\phi \in C^{2}(D)$ touches $f$ from below at $x_{0} \in D$, then there exists $x_{j} \rightarrow x_{0}$ such that

$$
f_{n_{l}}\left(x_{j}\right)-\phi\left(x_{j}\right)=\min _{D}\left\{f_{n_{j}}-\phi\right\} .
$$


Theorem 5.8 The function $w_{\infty}$ is a viscosity solution of

$$
\begin{cases}\Delta_{\infty(x)}\left(\frac{u}{\|\nabla u\|_{\infty}}\right)=0 & \text { in } \quad D=\Omega \backslash\left\{x_{\star}\right\} \\ \frac{u}{\|\nabla u\|_{\infty}}=d & \text { on } \quad \partial D=\partial \Omega \cup\left\{x_{\star}\right\} .\end{cases}
$$

Proof. Taking into account that $\left\|\nabla w_{\infty}\right\|_{\infty}=\Lambda_{\infty}$ we just need to show that $w_{\infty}$ satisfies

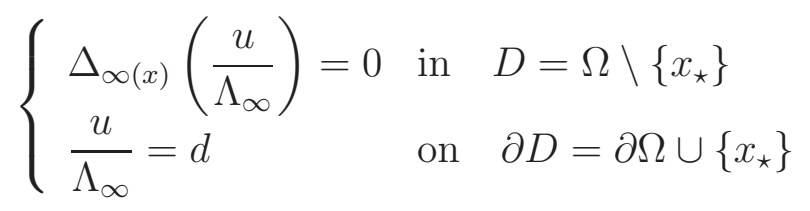

in the viscosity sense.

Since $\Lambda_{\infty}=\frac{1}{\|d\|_{\infty}}$, it follows from Corollary 5.3 that

$$
\frac{w_{\infty}\left(x_{\star}\right)}{\Lambda_{\infty}}=w_{\infty}\left(x_{\star}\right)\|d\|_{\infty}=d\left(x_{\star}\right) .
$$

Thus, taking into account that $\left.w_{\infty}\right|_{\partial \Omega}=0=\left.d\right|_{\partial \Omega}$, we conclude that $\frac{w_{\infty}}{\Lambda_{\infty}}=d$ on $\partial \Omega \cup\left\{x_{\star}\right\}$.

In order to show that $w_{\infty}$ is a viscosity supersolution, let $x_{0} \in \Omega \backslash\left\{x_{\star}\right\}$ and $\phi \in C^{2}\left(\Omega \backslash\left\{x_{\star}\right\}\right)$ be such that $\phi$ touches $w_{\infty}$ from below at $x_{0}$, i.e.

$$
\phi\left(x_{0}\right)=w_{\infty}\left(x_{0}\right) \text { and } \phi(x)<w_{\infty}(x) \text {, for } x \neq x_{0} .
$$

We claim that

$$
\Delta_{\infty(x)}\left(\frac{\phi\left(x_{0}\right)}{\Lambda_{\infty}}\right) \leq 0,
$$

where the expression of the differential operator is given by (38). Since the above inequality holds trivially when $\nabla \phi\left(x_{0}\right)=0$, we assume that $\left|\nabla \phi\left(x_{0}\right)\right| \neq 0$. So, let us take a ball $B_{\epsilon}\left(x_{0}\right) \subset \Omega \backslash\left\{x_{\star}\right\}$ such that

$$
|\nabla \phi(x)| \neq 0 \quad \text { in } B_{\epsilon}\left(x_{0}\right)
$$

Proposition 5.2 and its Corollary 5.3 guarantee the existence of a subsequence of indexes $\left(l_{n}\right)_{n \in \mathbb{N}}$ such that $w_{l_{n}} \rightarrow w_{\infty}$ in $C(\bar{\Omega})$ and $x_{0}^{l_{n}} \rightarrow x_{\star}$, where $x_{0}^{l_{n}}$ denotes a maximum point of $w_{l_{n}}$. It follows that $w_{l_{n}} \rightarrow w_{\infty}$ uniformly in $\overline{B_{\epsilon}\left(x_{0}\right)}$ and $x_{0}^{l_{n}} \notin B_{\epsilon}\left(x_{0}\right)$ for all $n$ large enough.

Applying Lemma 5.7 to $\overline{B_{\epsilon}\left(x_{0}\right)}$ we can assume that (up to pass to another subsequence) there exists $y_{n} \rightarrow x_{0}$ such that

$$
m_{n}:=\min _{B_{\epsilon}\left(x_{0}\right)}\left\{w_{l_{n}}-\phi\right\}=w_{l_{n}}\left(y_{n}\right)-\phi\left(y_{n}\right) .
$$

Thus, the function $\phi_{n}(x):=\phi(x)+m_{n}-\left|x-y_{n}\right|^{4}$, which belongs to $C^{2}\left(B_{\epsilon}\left(x_{0}\right)\right)$, satisfies

$$
\phi_{n}\left(y_{n}\right)=\phi\left(y_{n}\right)+m_{n}=w_{l_{n}}\left(y_{n}\right) \quad \text { and } \quad \phi_{n}(x) \leq w_{l_{n}}(x)-\left|x-y_{n}\right|^{4}<w_{l_{n}}(x)
$$


for all $x \in B_{\epsilon}\left(x_{0}\right) \backslash\left\{y_{n}\right\}$. That is, $\phi_{n}$ touches $w_{l_{n}}$ from below at $y_{n}$.

Let $H_{l}$ denote the differential operator associated with the equation $\Delta_{l p(x)}\left(u / \mu_{l}\right)=0$, that is,

$$
H_{l}\left(x, u, \nabla u, D^{2} u\right):=|\nabla u|^{l p(x)-4}\left\{|\nabla u|^{2} \Delta \phi+(l p(x)-2) \Delta_{\infty} u+|\nabla u|^{2} \ln \left(\frac{|\nabla u|}{\mu_{l}}\right)\langle\nabla u, l \nabla p\rangle\right\} .
$$

(Recall that $\mu_{l}=\left\|\nabla w_{l}\right\|_{l p(x)} \rightarrow \Lambda_{\infty}$ as $l \rightarrow \infty$.)

It follows from Proposition 5.6 that $w_{l}$ is a viscosity (super)solution of the equation

$$
H_{l}\left(x, u, \nabla u, D^{2} u\right)=0 .
$$

Hence, taking $\phi_{n}$ as a test function for $w_{l_{n}}$, it follows that

$$
H_{l_{n}}\left(y_{n}, \phi_{n}\left(y_{n}\right), \nabla \phi_{n}\left(y_{n}\right), D^{2} \phi_{n}\left(y_{n}\right)\right) \leq 0 \text {. }
$$

This means that

$$
\begin{aligned}
\left|\nabla \phi_{n}\left(y_{n}\right)\right|^{l_{n} p(x)-4}\left\{\left|\nabla \phi_{n}\left(y_{n}\right)\right|^{2} \Delta \phi_{n}\left(y_{n}\right)+\left(l_{n} p\left(y_{n}\right)-2\right) \Delta_{\infty} \phi_{n}\left(y_{n}\right)\right. & \\
& \left.+\left|\nabla \phi_{n}\left(y_{n}\right)\right|^{2} \ln \left(\left|\nabla \phi_{n}\left(y_{n}\right)\right| / \mu_{l_{n}}\right)\left\langle\nabla \phi_{n}\left(y_{n}\right), l_{n} \nabla p\left(y_{n}\right)\right\rangle\right\} \leq 0 .
\end{aligned}
$$

Since

$$
\nabla \phi_{n}\left(y_{n}\right)=\nabla \phi\left(y_{n}\right), \quad \Delta \phi_{n}\left(y_{n}\right)=\Delta \phi\left(y_{n}\right) \quad \text { and } \quad \Delta_{\infty} \phi_{n}\left(y_{n}\right)=\Delta_{\infty} \phi\left(y_{n}\right)
$$

and $\nabla \phi\left(y_{n}\right) \neq 0$ for $n$ large enough, we have

$$
\begin{aligned}
\left|\nabla \phi\left(y_{n}\right)\right|^{l_{n} p(x)-4}\left\{\left|\nabla \phi\left(y_{n}\right)\right|^{2} \Delta \phi\left(y_{n}\right)+\left(l_{n} p\left(y_{n}\right)-2\right) \Delta_{\infty} \phi\left(y_{n}\right)\right. & \\
& \left.+\left|\nabla \phi\left(y_{n}\right)\right|^{2} \ln \left(\left|\nabla \phi\left(y_{n}\right)\right| / \mu_{l_{n}}\right)\left\langle\nabla \phi\left(y_{n}\right), l_{n} \nabla p\left(y_{n}\right)\right\rangle\right\} \leq 0 .
\end{aligned}
$$

Dividing this inequality by $\left(l_{n} p\left(y_{n}\right)-2\right)\left|\nabla \phi\left(y_{n}\right)\right|^{l_{n} p(x)-4}$, we obtain

$$
\frac{\left|\nabla \phi\left(y_{n}\right)\right|^{2} \Delta \phi\left(y_{n}\right)}{l_{n} p\left(y_{n}\right)-2}+\Delta_{\infty} \phi\left(y_{n}\right)+\left|\nabla \phi\left(y_{n}\right)\right|^{2} \ln \left(\frac{\left|\nabla \phi\left(y_{n}\right)\right|}{\mu_{l_{n}}}\right)\left\langle\nabla \phi\left(y_{n}\right), \frac{\nabla p\left(y_{n}\right)}{p\left(y_{n}\right)-2 / l_{n}}\right\rangle \leq 0 .
$$

Then, by making $n \rightarrow \infty$ we arrive at

$$
\Delta_{\infty} \phi\left(x_{0}\right)+\left|\nabla \phi\left(x_{0}\right)\right|^{2} \ln \left(\frac{\left|\nabla \phi\left(x_{0}\right)\right|}{\Lambda_{\infty}}\right)\left\langle\nabla \phi\left(x_{0}\right), \frac{\nabla p\left(x_{0}\right)}{p\left(x_{0}\right)}\right\rangle \leq 0 .
$$

According to (38) this implies that

$$
\Delta_{\infty(x)}\left(\frac{\phi\left(x_{0}\right)}{\Lambda_{\infty}}\right) \leq 0
$$

and we conclude thus the proof that $w_{\infty}$ is a viscosity supersolution.

Analogously, we can show that if $\psi \in C^{2}\left(\Omega \backslash\left\{x_{\star}\right\}\right)$ touches $w_{\infty}$ from above at the point $x_{0}$, then

$$
\Delta_{\infty(x)}\left(\frac{\psi\left(x_{0}\right)}{\Lambda_{\infty}}\right) \geq 0 .
$$

Therefore, $w_{\infty}$ satisfies (39) in the viscosity sense.

The following uniqueness result can be found in [21]. 
Proposition 5.9 ( [21], Theorem 1.2) Let $D$ be a bounded domain of $\mathbb{R}^{N}$ and $f: \partial D \rightarrow \mathbb{R}$ be a Lipschitz continuous function. There exists a unique viscosity solution $u \in C(\bar{D}) \cap W^{1, \infty}(D)$ for the Dirichlet boundary value problem

$$
\begin{cases}\Delta_{\infty(x)} u=0 & \text { in } D \\ u=f & \text { on } \partial D\end{cases}
$$

It follows from this result, with $f=d$ and $D=\Omega \backslash\left\{x_{\star}\right\}$, that $w_{\infty}$ is the only solution of the Dirichlet problem (39).

Following the ideas of [31] and [8] we give a condition on $\Omega$ that leads to the equality $w_{\infty}=$ $d /\|d\|_{\infty}$. For this we recall that $d \in C^{1}(\Omega \backslash \mathcal{R}(\Omega))$ where $\mathcal{R}(\Omega)$ denotes the ridge of $\Omega$, defined as the set of all points in $\Omega$ whose distance to the boundary is reached at least at two points (see [4,9]). Notice that $\mathcal{R}(\Omega)$ contains the maximum points of $d$. Since $d$ is a viscosity solution of the eikonal equation $|\nabla u|=1$ in $\Omega$, it is simple to check that $\Delta_{\infty(x)} d=0$ in $\Omega \backslash \mathcal{R}(\Omega)$ in the viscosity sense.

Proposition 5.10 If $\mathcal{R}(\Omega)$ is a singleton set, then $w_{l} \rightarrow \frac{d}{\|d\|_{\infty}}$ uniformly in $\bar{\Omega}$ and $x_{0}^{l} \rightarrow x_{\star}$.

Proof. It follows from Corollary 5.3 that $\mathcal{R}(\Omega)=\left\{x_{\star}\right\}$, since $x_{\star}$ is a maximum point of $d$. Therefore, $d \in C^{1}\left(\Omega \backslash\left\{x_{\star}\right\}\right)$ what implies that $d$ is a viscosity solution of

$$
\begin{cases}\Delta_{\infty(x)} u=0 & \text { in } \Omega \backslash\left\{x_{\star}\right\} \\ u=d & \text { on } \partial\left(\Omega \backslash\left\{x_{\star}\right\}\right)=\partial \Omega \cup\left\{x_{\star}\right\}\end{cases}
$$

Therefore, by the uniqueness stated in Proposition 5.9 (with $D:=\Omega \backslash\left\{x_{\star}\right\}$ ) we have $\frac{w_{\infty}}{\Lambda_{\infty}}=d$, that is

$$
w_{\infty}=\frac{d}{\|d\|_{\infty}} \text { in } \bar{\Omega}
$$

These arguments imply that $d /\|d\|_{\infty}$ is the only limit function of any uniformly convergent subsequence of $\left(w_{l}\right)_{l \in N}$ and also that $x_{\star}$ is the only cluster point of the numerical sequence $\left(x_{0}^{l}\right)_{l \in N}$.

Balls, ellipses and other symmetric sets are examples of domains whose ridge is a singleton set.

\section{Acknowledgements}

C. O. Alves was partially supported by CNPq/Brazil (304036/2013-7) and INCT-MAT. G. Ercole was partially supported by CNPq/Brazil (483970/2013-1 and 306590/2014-0) and Fapemig/Brazil (APQ-03372-16). 


\section{References}

[1] E. Acerbi, G. Mingione, Regularity results for stationary electrorheological fluids, Arch. Rational Mech. Anal. 164 (2002), 213-259.

[2] C.O. Alves, J.L.P. Barreiro, Existence and multiplicity of solutions for a $p(x)$-Laplacian equation with critical growth, J. Math. Anal. Appl. 403 (2013), 143-154.

[3] S.N. Antontsev, J.F. Rodrigues, On stationary thermo-rheological viscous flows, Ann. Univ. Ferrara Sez. VII Sci. Mat. 52 (2006) 19-36.

[4] T. Bhatthacharya, E. Dibenedetto and J. Manfredi, Limits as $p \rightarrow \infty$ of $\Delta_{p} u_{p}=f$ and related extremal problems, Rendiconti del Sem. Mat., Fascicolo Speciale Non Linear PDE's, Univ. Torino (1989) 15-68.

[5] A. Chambolle, P.L. Lions, Image recovery via total variation minimization and related problems, Numer. Math. 76 (1997) 167-188.

[6] Y. Chen, S. Levine, M. Rao, Variable exponent, linear growth functionals in image restoration, SIAM J. Appl. Math. 66 (2006) 1383-1406.

[7] L. Diening, P. Harjulehto, P. Hästö, M. Rüžička, Lebesgue and Sobolev spaces with variable exponents, Springer, 2011.

[8] G. Ercole, G. Pereira, Asymptotics for the best Sobolev constants and their extremal functions, Math.Nachr. 289 (2016) 1433-1449.

[9] W.D. Evans, D.J. Harris, Sobolev embeddings for generalized ridge domains, Proc. London Math. Soc. 54 (1987) 141-175.

[10] X. Fan, Global $C^{1, \alpha}$ regularity for variable exponent elliptic equations in divergence form, J.Differential Equations 235 (2007) 397-417.

[11] X. Fan, On the sub-supersolution method for $p(x)$-Laplacian equations, J. Math. Anal. Appl. 330 (2007) 665-682.

[12] X. Fan, Remarks on eigenvalue problems involving the $p(x)$-Laplacian, J. Math. Anal. Appl. 352 (2009) 85-98.

[13] X. Fan, J. Shen, D. Zhao, Sobolev embedding theorems for spaces $W^{k, p(x)}$, J. Math. Anal. Appl. 262 (2001) 749-760.

[14] X. Fan, Q. Zhang, Existence of solutions for $p(x)$-Laplacian Dirichlet problems, Nonlinear Anal. 52 (2003) 1843-1852.

[15] X. Fan, D. Zhao, A class of De Giorgi type and Hölder continuity, Nonlinear Anal. 36 (1999) 295-318. 
[16] X. Fan, D. Zhao, On the spaces $L^{p(x)}(\Omega)$ and $W^{m, p(x)}(\Omega)$, J. Math. Anal. Appl. 263 (2001) 424-446.

[17] J. Fernández Bonder, N. Saintier, A. Silva, On the Sobolev embedding theorem for variable exponent spaces in the critical range, J. Differential Equations 253 (2012) 1604-1620.

[18] G. Franzina, P. Lindqvist, An eigenvalue problem with variable exponents, Nonlinear Analysis 85 (2013) 1-16.

[19] R. Hynd, E. Lindgren, Extremal functions for Morrey's inequality in convex domains, arXiv:1609.08186 [math.AP].

[20] P. Juutinen, P. Lindqvist, and J. Manfredi, The $\infty$-eigenvalue problem, Arch. Ration. Mech. Anal. 148 (1999) 89-105.

[21] P. Lindqvist, A curious equation involving the $\infty$-Laplacian, Advances in Calculus of Variations 3 (2010) 409-421.

[22] P. Lindqvist, Notes on the infinity Laplace equation, Springer, 2016.

[23] M. Mihăilescu, V. Rădulescu, On a nonhomogeneous quasilinear eigenvalue problem in Sobolev spaces with variable exponent, Proc. Amer. Math. Soc. 135 (2007) 2929-2937.

[24] Y. Mizuta, T. Ohno, T. Shimomura, N. Shioji, Compact embeddings for Sobolev spaces of variable exponents and existence of solutions for nonlinear elliptic problems involving the $p(x)$-Laplacian and its critical exponent, Ann. Acad. Sci. Fenn. Math. 35 (2010) 115-130.

[25] K. Perera, M. Squassina, Asymptotic behavior of the eigenvalues of the $p(x)$-Laplacian, Manuscripta Math. 144 (2014) 535-544.

[26] V. Rădulescu, Nonlinear elliptic equations with variable exponent: old and new, Nonlinear Anal. 121 (2015) 336-369.

[27] V. Rădulescu, D. Repovš, Partial differential equations with variable exponents. Variational methods and qualitative analysis. Monographs and Research Notes in Mathematics. CRC Press, Boca Raton, FL, 2015.

[28] M. Ruzicka, Electrorheological fluids: Modeling and mathematical theory. Lecture Notes in Math., vol. 1748, Springer-Verlag, Berlin (2000).

[29] I. Stancut, I. Stircu, Eigenvalue problems for anisotropic equations involving a potential on Orlicz Sobolev type spaces, Opuscula Math. 36 (2016) 81-101.

[30] A. Szulkin, Ljusternik Schnirelmann theory on $C^{1}$ manifolds, Ann. Ins. H. Poincaré Anal. Non Linéaire 5 (1988) 119-139.

[31] Y. Yu, Some properties of the ground states of the infinity Laplacian, Indiana Univ. Math. J. 56 (2007) 947-964. 